ARTICLE

\title{
HOXBLINC long non-coding RNA activation promotes leukemogenesis in NPM1-mutant acute myeloid leukemia
}

Ganqian Zhu1,2,19, Huacheng Luo ${ }^{3,19}$, Yang Feng ${ }^{4}$, Olga A. Guryanova (1) 4, Jianfeng Xu ${ }^{5}$, Shi Chen 1,2, Qian Lai (1) ${ }^{3}$, Arati Sharma ${ }^{6}$, Bing $\mathrm{Xu}^{7}$, Zhigang Zhao ${ }^{8}$, Ru Feng ${ }^{9}$, Hongyu Ni ${ }^{10}$, David Claxton ${ }^{6,11}$, Ying Guo ${ }^{2,12}$, Ruben A. Mesa ${ }^{13,14}$, Yi Qiu (1) 11,15, Feng-Chun Yang 2,12,14,16, Wei Li (1) ${ }^{17}$, Stephen D. Nimer ${ }^{16,18,}$ Suming Huang (1) $3,11,20 \times$ \& Mingjiang Xu (1) 1,2,14,16,20凶

Nucleophosmin (NPM1) is the most commonly mutated gene in acute myeloid leukemia (AML) resulting in aberrant cytoplasmic translocation of the encoded nucleolar protein $\left(\mathrm{NPM1c}^{+}\right)$. NPM1c ${ }^{+}$maintains a unique leukemic gene expression program, characterized by activation of HOXA/B clusters and MEIS1 oncogene to facilitate leukemogenesis. However, the mechanisms by which $\mathrm{NPM1c}^{+}$controls such gene expression patterns to promote leukemogenesis remain largely unknown. Here, we show that the activation of HOXBLINC, a HOXB locus-associated long non-coding RNA (IncRNA), is a critical downstream mediator of NPM1c ${ }^{+}$-associated leukemic transcription program and leukemogenesis. HOXBLINC loss attenuates NPM1c ${ }^{+}$-driven leukemogenesis by rectifying the signature of $\mathrm{NPM1c}^{+}$leukemic transcription programs. Furthermore, overexpression of HoxBlinc (HoxBlincTg) in mice enhances HSC self-renewal and expands myelopoiesis, leading to the development of AMLlike disease, reminiscent of the phenotypes seen in the $\mathrm{Npm} 1$ mutant knock-in $\left(\mathrm{Npm}^{\mathrm{C} /+}{ }^{\mathrm{C}}\right.$ ) mice. HoxBlincTg and $\mathrm{Npm}^{\mathrm{C} /}+\mathrm{HSPCs}$ share significantly overlapped transcriptome and chromatin structure. Mechanistically, HoxBlinc binds to the promoter regions of NPM1c ${ }^{+}$ signature genes to control their activation in HoxBlincTg HSPCs, via MLL1 recruitment and promoter H3K4me3 modification. Our study reveals that HOXBLINC IncRNA activation plays an essential oncogenic role in NPM1c ${ }^{+}$leukemia. HOXBLINC and its partner MLL1 are potential therapeutic targets for $\mathrm{NPM}^{+} \mathrm{c}^{+} \mathrm{AML}$. 
N ucleophosmin (NPM1) mutations are the most frequently recurring genetic abnormalities in patients with acute myeloid leukemia (AML), occurring in approximately $50 \%$ of adults and $20 \%$ of childhood AML with normal karyotypes ${ }^{1,2}$. NPM1-mutated AML has been included as a distinct AML entity in the World Health Organization (WHO) classification ${ }^{3}$. NPM1 encodes a protein that is normally located in the nucleolus and has multiple functions such as biogenesis of ribosomes and maintenance of genomic stability ${ }^{4}$. NPM1 mutations result in cytoplasmic mislocalization of the mutant protein $\left(\mathrm{NPM}_{1}{ }^{+}\right)$, which is critical for its role in leukemogenesis ${ }^{5,6}$. As an AML-initiating lesion, $\mathrm{NPMlc}^{+}$maintains a distinctive transcriptional signature in AML cells, characterized by upregulation of HOXA and HOXB cluster genes and their oncogenic cofactor $M E I S 1^{7,8}$. However, the precise mechanisms by which NPM1c ${ }^{+}$ drives the leukemic gene expression programs remain unclear.

Dysregulation of $H O X A / B$ genes is a dominant mechanism of leukemic transformation and hematopoietic stem/progenitor cell (HSPC) deregulation ${ }^{9}$. A wide variety of molecular determinants including transcription factors, epigenetic regulators (e.g., polycomb and trithorax proteins), microRNAs, chromatin structure, and long non-coding RNAs (lncRNAs) are known to control $H O X$ gene expression. However, their relationship with each other to fine-tune the $H O X A / B$ gene expression pattern in AML remains to be elucidated. Recently two $H O X A / B$ loci associated lncRNAs, HOTTIP and HOXBLINC, were shown to regulate transcription of $H O X A / B$ genes through influencing epigenetic landscape ${ }^{10-12}$. HOXBLINC has been reported to play a critical role in hematopoietic specification during development through its cis-acting function to coordinate anterior $H O X B$ gene expression via recruitment of the SETD1A/MLL1 histone H3K4 methyltransferase complexes ${ }^{10}$.

Located in the anterior $H O X B$ locus and serving as a regulator of HOXB gene transcription, the role of HOXBLINC in HSPC biology and leukemogenesis remains unknown. In this study, we demonstrate that HOXBLINC is upregulated restrictively in $\mathrm{NPM}_{1} \mathrm{c}^{+}$AML. Npm1 mutant knock-in $\left(\mathrm{Npm}^{\mathrm{c} /+}\right)$ and HoxBlincTg HSPCs share significantly overlapping chromatin signatures and gene expression profiles in their upregulated genes as compared to WT HSPCs, including the NPMlc ${ }^{+}$signature $H o x A / B$ cluster genes and homeobox oncogene Meis1. Transgenic overexpression of HoxBlinc lncRNA in hematopoietic cells led to the development of an AML-like disease by triggering HSC selfrenewal and expanding myelopoiesis, similar to the phenotypes displayed by $N p m 1^{\mathrm{c} /+}$ mice, while inhibition of HOXBLINC in $\mathrm{NPM}^{+}{ }^{+}$AML cells mitigates leukemogenesis. Importantly, HoxBlinc overexpression in HSPCs increases its binding to $\mathrm{NPM}^{+} \mathrm{c}^{+}$signature genes and drives the leukemic specific transcription program in HSPCs by recruiting the MLL1 complex to reorganize local chromatin signatures. Together, our studies provide compelling evidence for the potent oncogenic role of HOXBLINC in NPM1c ${ }^{+}$-mediated leukemogenesis. HOXBLINC lncRNA and MLL1 could serve as potential therapeutic targets for the treatment of NPM1c $c^{+}$AML.

\section{Results}

HOXBLINC is specifically upregulated in NPMIc ${ }^{+}$AML. Upregulation of $H O X$ genes, especially $H O X A$ and $H O X B$ cluster genes are not only a characteristic but also a dominant mechanism for the pathogenesis of $\mathrm{AML}^{9}$. HoxBlinc lncRNA has been shown to be required to activate anterior $\operatorname{HoxB}$ gene transcription during development ${ }^{10}$. To determine whether HOXBLINC is aberrantly expressed along with the HOXB genes in AML, we performed RTqPCR on bone marrow mononuclear cells (BMMNCs) from a cohort of AML patients $\left(N P M 1 c^{+}, n=25\right.$; and NPM1-wt, $n=40$; see patient information in Supplementary Table 1) as compared to both BMMNCs $(n=16)$ and $\mathrm{CD} 34^{+}$cells $(n=11)$ from healthy individuals. Interestingly, a dramatic upregulation of HOXBLINC was observed specifically in NPM1c ${ }^{+}$AML patients (Fig. 1a) as compared to NPM1-wt patients and normal $\mathrm{CD} 34^{+}$cells. When the RNA-seq data from TCGA-LAML datasets consisting of a cohort of 181 AML patients was analyzed for HOXBLINC expression, significantly higher HOXBLINC expression was observed in $N P M 1 c^{+}$, but not $M L L$-rearranged $\left(M L L r^{+}\right)$AML patients as compared to NPM1c ${ }^{-} M L L r^{-}$patients (Fig. 1b). The expression of HOXBLINC was positively correlated with the expression levels of NPM1c ${ }^{+}$ signature genes including anterior HOXB genes, HOXA9 and MEIS1, but not HOXB13 in this AML cohort (Supplementary Fig. 1a). Interestingly, AML patients with high HOXBLINC expression (the top thirty percentile of patients) had a significantly shortened survival as compared to patients with low HOXBLINC expression (the bottom thirty percentile, Supplementary Fig. 1b). Consistently, HOXBLINC was highly expressed in NPM1c ${ }^{+}$OCIAML3 and IMS-M2 AML cells, but not NPM1-wt AML cells such as MLLr' MOLM-13, MV4-11, THP-1, NOMO-1, and OCI-AML2 cells, as well as BCR-ABL1 ${ }^{+}$K562 and JAK2V617F+ SET-2 cells (Supplementary Fig. 1c). These data collectively indicate that HOXBLINC is upregulated specifically in NPM1c ${ }^{+} \mathrm{AML}$ patients.

Loss of HOXBLINC perturbs NPM1c ${ }^{+}$-mediated transcription programs and leukemogenesis. To confirm HOXBLINC activation is a downstream event of NPM1c ${ }^{+}$and determine the role of HOXBLINC in NPM1c ${ }^{+}$-mediated transcription regulation and leukemogenesis, we performed RNA-seq analysis on LSK cells isolated from the Npm1 mutant knock-in $\left(\mathrm{Npm}^{\mathrm{c} /+}\right) \mathrm{mice}^{13}$. As compared to WT LSK cells, $N p m 1^{c /+}$ LSK cells had 871 downregulated genes and 980 upregulated genes, including HoxBlinc and the NPM1c ${ }^{+}$signature genes HoxB2-5, HoxA7,9-11, Meis1, and Runx1 (Fig. 1c, Supplementary Fig. 1d), some of which are confirmed by qPCR (Supplementary Fig. 1e). Gene Ontology (GO) and gene set enrichment (GSEA) analyses revealed that the upregulated genes in $\mathrm{Npm}^{c /+}$ vs. WT LSK cells are enriched with cell fate commitment, cell cycle, myeloid cell proliferation, stem cell maintenance, Wnt and Jak-STAT signaling pathways, pathways in cancer, as well as AML NPM1-mutated and HOXA9 oncogenic pathway (Supplementary Fig. 1f, g).

We next examined the effect of HOXBLINC loss on NPM1c ${ }^{+}$mediated transcription regulation and leukemogenesis using NPM1c ${ }^{+}$OCI-AML3 cells. We created CRISPR-dCas9KRAB mediated HOXBLINC epigenetic silencing clones (HOXBLINCi) by targeting the KRAB repressive domain to the HOXBLINC promoter (Supplementary Fig. 2a). We then compared genome-wide transcriptome changes between control and HOXBLINCi OCI-AML3 cells by performing RNA-seq analysis. Consistently, NPM1c $\mathrm{c}^{+}$OCI-AML3 cells exhibited high expression of HOXBLINC lncRNA and the common NPM1c ${ }^{+}$ AML signature genes (Fig. 1d). Interestingly, inhibition of HOXBLINC in OCI-AML3 cells significantly impaired the transcription of many $\mathrm{NPM}^{+} \mathrm{c}^{+}$signature genes such as HOXB2-5, HOXA9-11, RUNX1, and MEIS1 (Fig. 1d). GSEA and GO analyses revealed that loss of HOXBLINC affects the pathways and genes involved in AML with NPM1-mutated, HOXA9 pathway, pathways in cancer, cell cycle, cell fate commitment, myeloid cell differentiation, and Wnt and JAKSTAT signaling pathways (Fig. 1e, f; Supplementary Fig. 2b), similar to those observed in the upregulated genes of $\mathrm{Npm}^{\mathrm{c} /+}$ vs. WT LSK cells (Supplementary Fig. 1f, g).

We further explored whether HoxBlinc is required for $\mathrm{NPM} 1 \mathrm{c}^{+}$-mediated leukemogenesis by generating two Tet-ON 

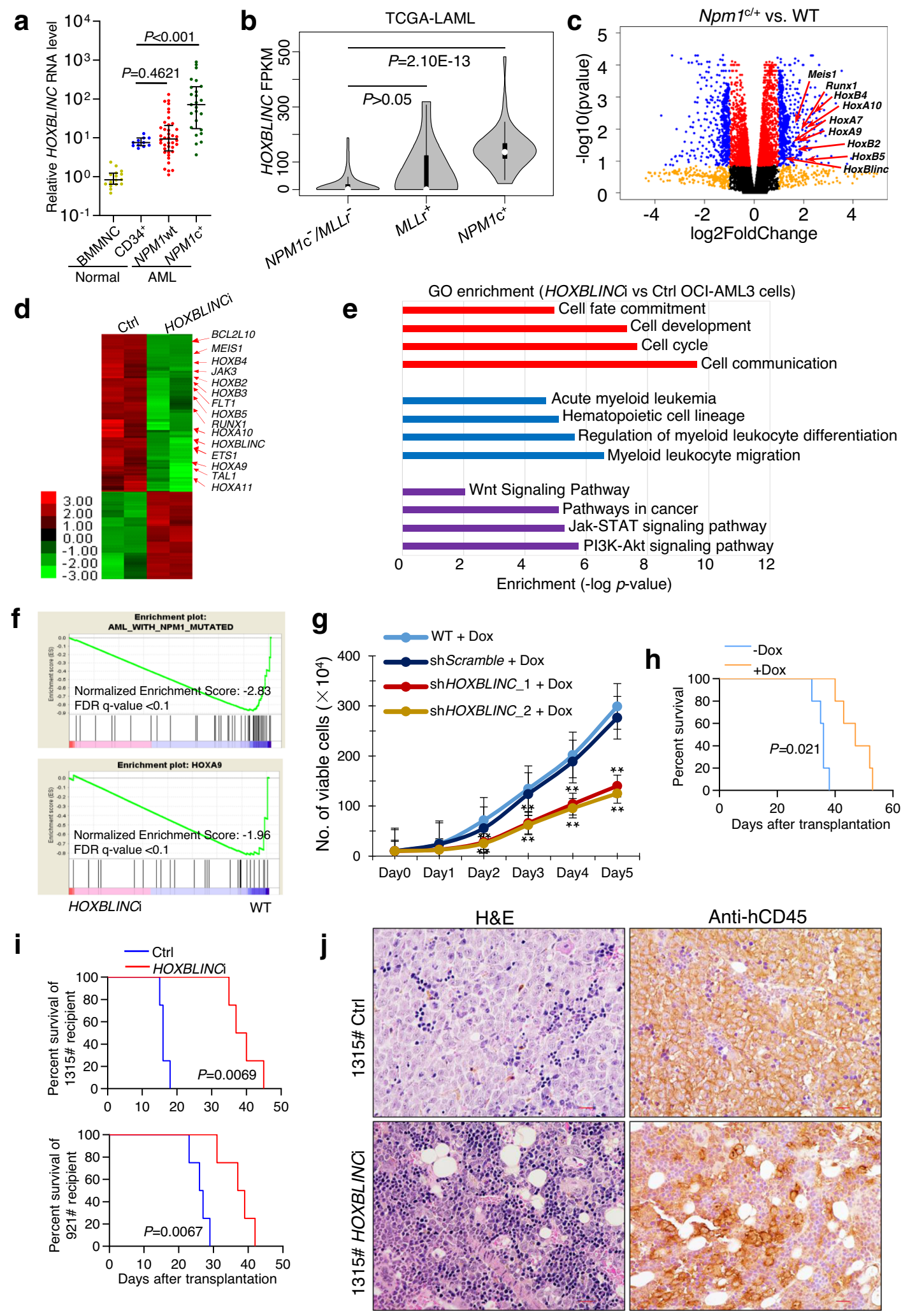

inducible HOXBLINC shRNA knockdown (KD) OCI-AML3 clones (Supplementary Fig. 2c). HOXBLINC KD significantly impaired OCI-AML3 cell proliferation compared to the scramble control (Fig. 1g). Cell cycle analysis revealed that HOXBLINC KD increased the sub-G0 cell population, suggesting HOXBLINC perturbation induced apoptosis (Supplementary Fig. 2d). When we transplanted a
Dox-inducible HOXBLINC KD OCI-AML3 clone into NOD-scid IL2R $\gamma^{\text {null }}$ (NSG) mice $\left(2 \times 10^{5}\right.$ cells/mouse) followed by Dox induction or vehicle treatment, the Dox-treated recipient mice had significantly prolonged survival as compared to the recipients without Dox treatment (Fig. 1h). At 30 days after transplantation, Dox-treated mice had significantly lower $\mathrm{hCD} 45^{+}$chimerism in 
Fig. 1 HOXBLINC is activated in NPM1c ${ }^{+}$AML patients and loss of HOXBLINC perturbs NPM1c $^{+}$-mediated transcription program and leukemogenesis. a RT-qPCR analysis of HOXBLINC RNA expression in BMMNCs $(n=16)$ and/or CD34+ cells $(n=11)$ from healthy individuals and in BMMNCs from NPM1wt $(n=40)$ or NPM1c $(n=25)$ AML patients (Data is presented as Median \pm interquartile; $P$ value was calculated by two-sided Mann Whitney nonparametric test). b TCGA database (GSE62944) was used to retrieve RNA expression levels of HOXBLINC in BM cells from AML patients without $N P M 1$ mutations and MLL rearrangements (NPM1c $\left./ M L L r^{-}, n=128\right)$, AML patients with $M L L$ rearrangements $\left(M L L r^{+}, n=11\right)$, or AML patients with $N P M 1$ mutations (NPM1c ${ }^{+}, n=42$ ). Violin plots show mean, interquartile, and $1.5 x$ interquartile. The width shows the probability density. $P$ was calculated by two-tailed t-test. c Volcano plot of RNA-seq analysis of differentially expressed genes in Npmic ${ }^{+}$knock-in (Npmic/+) vs. WT LSK cells based on two independent experiments. d Heat map of RNA-seq analysis shows the up- and down-regulated genes in HOXBLINC-KRAB vs. WT OCI-AML3 cells based on two independent experiments. Arrows: dysregulated genes implicated in leukemogenesis. e The HOXBLINC-KRAB affected genes in OCI-AML3 cells were analyzed and annotated by the Gene Ontology (GO) analysis. f Enrichment of HOXBLINC-KRAB dysregulated genes in NPM1-mutated (top) and HOXA9 (bottom) oncogenic pathways by Gene Set Enrichment Analysis (GSEA). g Proliferation curves of WT, shScramble-expressing, and two different shHOXBLINC-expressing OCI-AML3 cells upon doxycycline (Dox) treatment for 5 days. (Bars represent the mean \pm SD of total viable cells from three independent experiments; two-tailed t-test; ${ }^{\star \star} P<0.01 ; n=3$ independent experiments). $\mathbf{h}$ Kaplan-Meier survival curve of inducible shHOXBLINC OCI-AML3 cell transplanted mice treated with or without Dox (Log-rank test; $n=5$ mice/group). i Kaplan-Meier survival curves of mice transplanted with control or HOXBLINC-KRAB (HOXBLINCi) BM cells from 2 AML patients with NPM1 mutation. 1315\# patient (NPM1c+;FLT3wt, left), 921\# patient (NPM1c+;FLT3mu, right). $n=4$ mice/group. The Log-rank test was used to analyze differences between the survival curves. j Representative images of H\&E (right) and anti-hCD45 immunostaining (left) of femur sections from 4 mice transplanted with control or HOXBLINCi BM cells from 1315\# patients for 18 days $(\mathrm{scale}=20 \mu \mathrm{m})$.

the $\mathrm{BM}$, spleen and peripheral blood $(\mathrm{PB})$ of the recipients compared to the untreated animals (Supplementary Fig. 2e). These results indicate that HOXBLINC KD suppresses OCI-AML3 leukemic cell proliferation both in vitro and in vivo, likely through the normalization of $\mathrm{NPMlc}^{+}$-induced abnormal gene expression patterns (Fig. 1d). In addition, we silenced HOXBLINC expression in primary AML cells with or without NPM1c ${ }^{+}$mutation (\#1315: NPM1c FLT3wt, \#921: NPM1c ${ }^{+} ;$FLT3mu, \#LPP4: NPM1wt;MLLr ${ }^{+}$) by the CRISPR-dCas9-KRAB (HOXBLINCi), and then xenografted them into NSG mice. Both \#1315 and \#921 exhibited high HOXBLINC expression, while \#LPP4 had low HOXBLINC expression (Supplementary Table 1). In line with cell line xenograft results, mice receiving HOXBLINCi AML cells with NPM1c ${ }^{+}$mutations (\#1315 and \#921) had significantly prolonged survival as compared to mice transplanted with control cells (Fig. 1i). HOXBLINCi dramatically decreased the $\mathrm{hCD} 45^{+}$cell chimera in BM, spleen, and $\mathrm{PB}$ of these recipients (Supplementary Fig. 2f-h). Mice receiving control \#1315 AML cells became moribund around 18 days after transplantation. Selected mice receiving either control or HOXBLINCi \#1315 AML cells were sacrificed 18 days after transplantation to examine their human AML cell engraftment and AML development by combined FACS, immunohistochemical and morphological (cytospin) analyses on their BM cells. The engrafted human cells $\left(\mathrm{hCD} 45^{+}\right)$in both control and HOXBLINCi recipients were positive for $\mathrm{hCD} 33$ and negative for $\mathrm{CD} 19$ and $\mathrm{CD} 3$, with a small fraction $(\sim 4 \%)$ being $\mathrm{CD} 34^{+} \mathrm{CD} 33^{\text {low }}$ and over $50 \%$ being $\mathrm{CD} 34^{\text {low } /+} \mathrm{CD} 33^{+}$(Supplementary Fig. 2f). Immunohistochemical analyses of BM sections also revealed robust infiltration by human $\mathrm{CD}^{4} 5^{+}$cells (Fig. 1j) and BM cytospins showed significant proportions of myeloid blasts (Supplementary Fig. $2 \mathrm{~g}$ ). These data demonstrated that the engraftment was from human AML cells but not multi-lineage human hematopoietic cells, and also clarified that moribund/death of these animals were caused by AML development and progression. In contrast, HOXBLINCi neither prolonged the survival nor decreased $\mathrm{hCD} 45^{+}$ cell chimera in mice transplanted with NPM1wt;MLLr+ (\#LPP4) AML cells (Supplementary Fig. 2i). Thus, HOXBLINC perturbation decreased tumor burden and attenuated leukemic progression in vivo likely specific for NPM1c ${ }^{+}$AML patients.

Transgenic expression of HoxBlinc in hematopoiesis leads to lethal AML-like disease in mice. It has been shown that activation of a humanized $\mathrm{NPM}^{+} \mathrm{c}^{+}$knock-in allele in mouse HSCs $\left(\mathrm{NPM1}^{\mathrm{c} /+}\right)$ causes Hox gene overexpression, enhanced self-real, and expanded myelopoiesis, as well as the development of delayed-onset $\mathrm{AML}^{14}$. Since NPM1c ${ }^{+}$activates HOXBLINC which is critical for

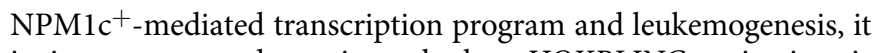
is important to determine whether HOXBLINC activation is sufficient to cause abnormal hematopoiesis and myeloid malignancies similar to the $N P M 1^{\mathrm{c} /+}$ mice. We first examined the HoxBlinc expression pattern along the HSC differentiation hierarchy. HoxBlinc expression was high in long-term (LT) and short-term (ST) HSCs, decreased in progenitor cells (MPP, CMP, and GMP), and was further decreased in the mature lineage cell populations except the $\mathrm{B} 220^{+} \mathrm{B}$ cells (Fig. 2a). The expression pattern of HoxBlinc in hematopoiesis suggests that this lncRNA might play an important role in regulating HSPC function. To investigate the impact of HoxBlinc activation on normal hematopoiesis and leukemogenesis in vivo, we generated a HoxBlinc transgenic $(\mathrm{Tg})$ mouse model in which full-length mouse HoxBlinc cDNA was inserted into the mouse genome under the control of Vav1 promoter and enhancer (HS321/45-vav vector) to ensure the expression of transgene specifically in hematopoiesis (Fig. 2b). Two founder HoxBlincTg mice were obtained. The transgene was inserted into the intron of the Bin1 gene on chromosome 18q for Tg Line \#1 (Fig. 2c). The expression levels of HoxBlinc RNA in BM cells were $~ 18$ - and 3-folds greater than the endogenous HoxBlinc expression in Tg Line \#1 and \#2, respectively (Fig. 2d).

Monitoring of a cohort of HoxBlincTg (Line \#1) mice showed that within 1 year of age, $67 \%$ of HoxBlincTg mice (10 of 15) died or were killed because of moribund conditions, whereas none of the WT mice $(n=12)$ died (Fig. 2e). Moribund HoxBlincTg mice exhibited weight loss, hepatosplenomegaly, enlarged lymph nodes as well as pale footpads and femurs as compared to WT (Fig. 2f, Supplementary Fig. 3a). Peripheral blood examination revealed marked leukocytosis due to elevated immature myeloid cells and neutrophils, thrombocytopenia, and severe anemia in these moribund HoxBlincTg mice (Supplementary Fig. 3b). Morphologically, May-Grünwald-Giemsa stained $\mathrm{PB}$ smears showed significantly increased blasts (Fig. 2g, left). BM cell cytospin preparations also demonstrated a predominance of myeloid cells with increased immature myeloid precursors (Fig. 2g, right). Flow cytometric analyses of the BM cells revealed increased c-Kit ${ }^{+}$ (consistently $>20 \%$ ) and Gr-1 low immature myeloid cell populations, as well as decreased lymphoid and erythroid cell populations (Supplementary Fig. 3c, d). Morphologic evaluation of $\mathrm{BM}$ histologic sections revealed myeloid hyperplasia with increased immature myeloid precursors, which were myeloperoxidase (MPO) positive indicating myeloid origin (Fig. $2 \mathrm{~h}$, Supplementary Fig. 3e). In addition, histologic evaluation of the 
a

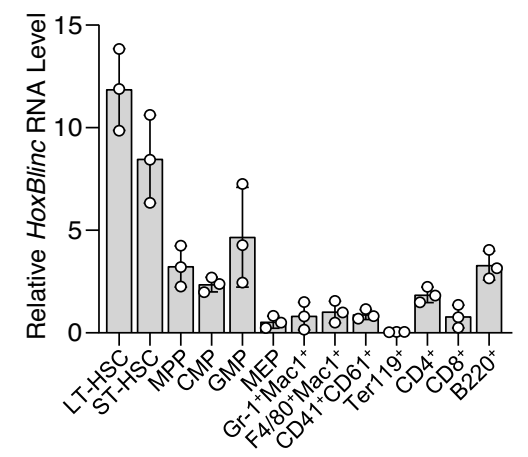

e

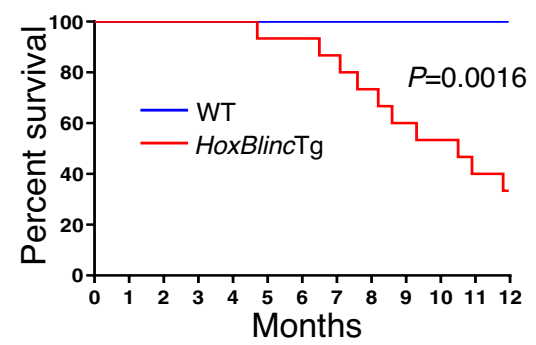

f

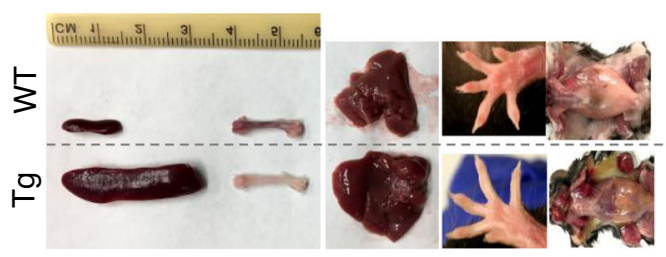

h

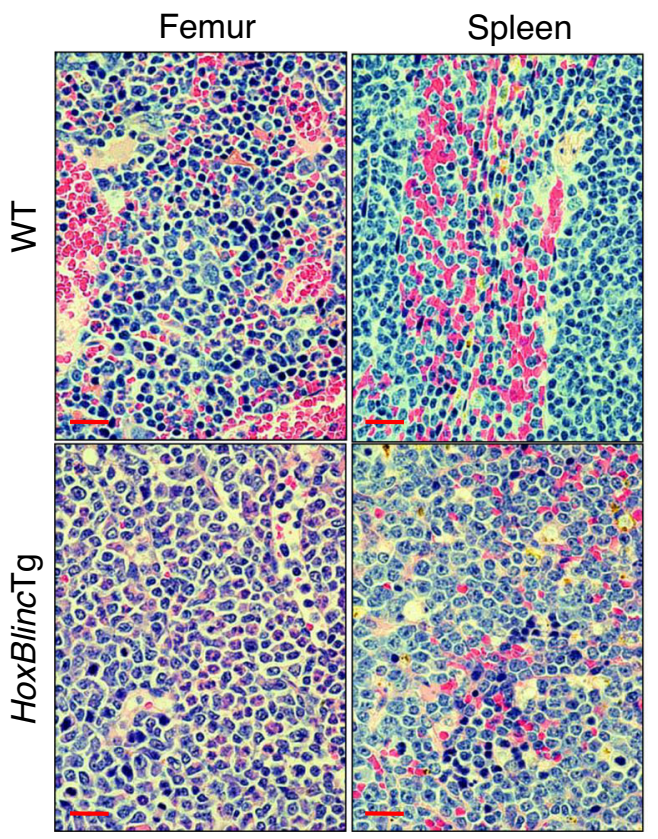

C b

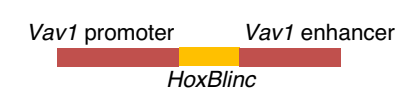

$$
\text { gin1 }-\frac{\text { exon6 }}{\text { HoxBlinc transgene }}
$$

\section{g}
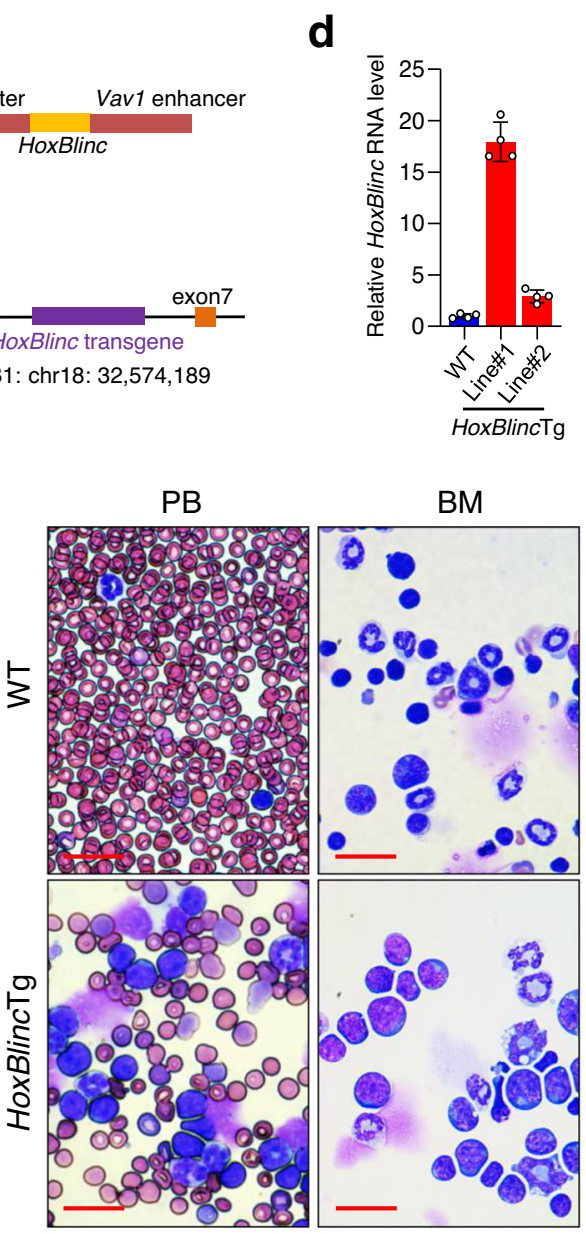

i

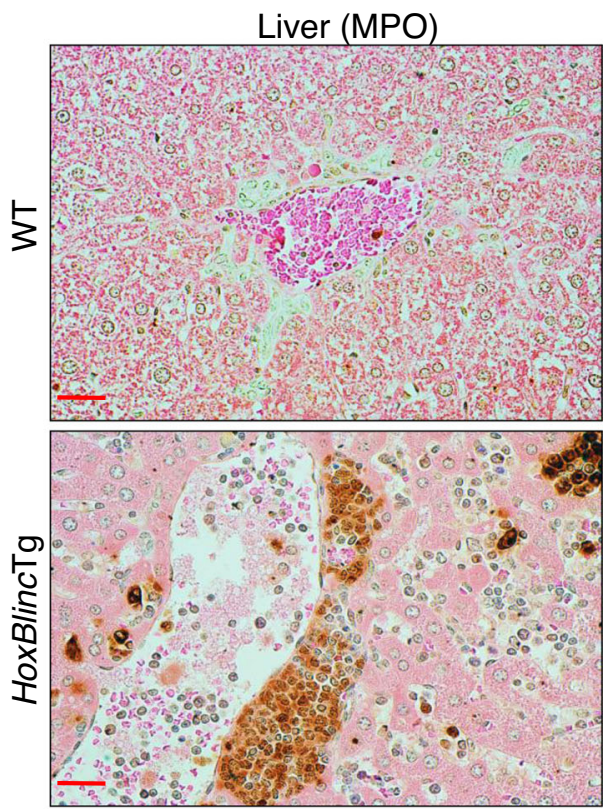

HoxBlincTg spleen, liver, and lymph node sections showed distortion of normal organ architecture with infiltration of MPO positive myeloid cells (Fig. 2h, i \& Supplementary Fig. 3f). The sections of liver demonstrated a sinusoidal infiltration pattern with clusters of immature myeloid cells (Fig. 2i, Supplementary Fig. 3f). Consistently, Line \#2 HoxBlincTg mice also developed
AML-like disease similar to Line \#1, characterized by shortened survival and $>20 \%{\mathrm{c}-\mathrm{Kit}^{+}}^{+}$myeloid cells in the $\mathrm{BM}, \mathrm{PB}$, and spleen (Supplementary Fig. 3g-i). The longer survival exhibited in Line \#2 than Line \#1 HoxBlincTg mice suggests a dosage effect of the HoxBlinc expression on hematopoiesis and transformation. These data demonstrate that similar to $N P M 1^{\mathrm{c} /+}$ mice, HoxBlinc 
Fig. 2 Transgenic overexpression of HoxBlinc in mice lead to lethal AML. a RT-qPCR analyses of HoxBlinc expression levels on FACS-sorted BM cell populations along the hematopoiesis hierarchical differentiation tree of WT mice. Data are presented as the mean \pm standard deviation (SD) of three independent experiments, each replicate utilized the flow-sorted populations of cells from combined BM cells of 7 WT mice. $\mathbf{b}$ Diagram of the Vav1 promoter/enhancer driven HoxBlinc transgene strategy. c PCR-based transgenic integrating location identification (TAIL) assay maps the Vav7-HoxBlinc transgene integration site to the mouse chromosome 18 in Line \#1 HoxBlincTg mice. d RT-qPCR analysis of the HoxBlinc RNA expression in BM cells of WT and 2 lines of HoxBlincTg mice. Data are presented as the mean \pm SD of four independent experiments. e Kaplan-Meier survival curve of WT ( $n=15)$ and HoxBlincTg (Line \#1, $n=15$ ) mice up to 1 year of age. $P$ value was calculated by Log-rank test. $\mathbf{f}$ Gross appearance of spleens, femur, livers, feet, and lymph nodes of representative WT and moribund HoxBlincTg mice. $\mathbf{g}$ Representative images of May-Giemsa stained PB smears and BM cytospins prepared from 6 WT and 6 moribund HoxBlincTg mice. h Representative images of H\&E stained femur and spleen sections of 6 WT and 6 moribund HoxBlincTg mice. i Representative images of MPO stained liver sections from 3 WT and 4 moribund HoxBlincTg mice. For panel $(\mathbf{g}-\mathbf{i})$, Scale $=20 \mu \mathrm{m}$.

overexpression in mice is sufficient to cause abnormal hematological characteristics resembling AML.

Transgenic expression of HoxBlinc enhances HSC self-renewal and expands myelopoiesis. The capacity of NPM1c ${ }^{+}$to promote enhanced HSC self-renewal and myeloid expansion has been well clarified $^{14}$. To further understand the role of HoxBlinc in AML pathogenesis, we then determined the effect of HoxBlinc overexpression on HSPC function. Flow cytometric analyses on BM cells of young HoxBlincTg mice (8-10 weeks, Line \#1) showed dramatically increased proportions of $\mathrm{Gr} 1^{+} / \mathrm{Macl}^{+}$granulocytic/ monocytic and $\mathrm{B} 220^{+} \mathrm{B}$ cells as well as decreased proportions of $\mathrm{CD}^{+} / \mathrm{CD}^{+} \mathrm{T}$ and Ter119+ erythroid cells as compared to agematched WT mice (Supplementary Fig. 4a). Importantly, HoxBlincTg BM cells contained a significantly greater proportion of Lin ${ }^{-}$Scal- ${ }^{+}{ }^{+}$- $\mathrm{Kit}^{+}(\mathrm{LSK})$ cells, while $\mathrm{Lin}{ }^{-} \mathrm{Scal}-1^{-} \mathrm{c}-\mathrm{Kit}^{+}(\mathrm{LK})$ cell population was similar as compared to WT mice (Fig. 3a). When the total number of LT-HSCs, ST-HSCs, and multipotent progenitor cells (MPPs) per femur were calculated based on their proportions within the LSK cell population and BM cellularity, the pools of both LT- and ST-HSCs, but not MPP were significantly expanded, although only the proportion of ST-HSCs within LSK cells were increased (Fig. 3b, Supplementary Fig. 4b). When each myeloid progenitor population was analyzed within the LK cells, a significantly higher percentage of GMP, but lower percentages of MEP/CMP cell populations were observed in HoxBlincTg mice than WT mice (Fig. 3c, Supplementary Fig. 4c). Consistent with the increased frequency of LSK and GMP, colony-forming unit cell (CFU-C) assays revealed significant higher frequencies of CFU-Cs, especially CFU-GM in the BM of HoxBlincTg mice than WT mice (Supplementary Fig. 4d). Similar to Line \#1, significantly increased proportions of $\mathrm{Gr} 1^{+} / \mathrm{Macl}^{+}$ and decreased proportions of erythroid cells were observed in the $\mathrm{BM}$ of Line \#2 HoxBlincTg mice as compared to WT mice (Supplementary Fig. 4e). Line \#2 HoxBlincTg BM cells also contained higher percentages of LSK and GMP cells than WT (Supplementary Fig. 4f, g). The similar hematological and disease phenotypes in both lines of HoxBlincTg mice indicate that these observed phenotypes are induced by the HoxBlinc transgenic expression but not the positional effect. Therefore, overexpression of HoxBlinc dysregulates HSPC pools with skewed hematopoiesis towards myeloid lineage in vivo.

We next examined the effect of HoxBlinc overexpression on the self-renewal and repopulation capacity of HSCs using in vitro replating and paired-daughter cell assays and in vivo competitive transplantation. A significantly higher replating potential was observed in each of the four successive rounds of replating in HoxBlincTg LSK cells than WT cells (Fig. 3d). Both symmetric and asymmetric cell divisions are required for the preservation of a normal HSC pool and continuous production of sufficient blood cells. Paired-daughter cell assays using WT and HoxBlinc Tg primitive CD34- LSK cells showed a higher proportion of HoxBlincTg CD34-LSK cells with symmetric self-renewal capacity, while the cells that underwent symmetric differentiation or asymmetric self-renewal were reduced as compared to WT (Fig. 3e). Competitive transplantation assays showed that the donor cell $\left(\mathrm{CD} 45.2^{+}\right)$chimerism in the $\mathrm{PB}$ of recipients transplanted with HoxBlincTg BM cells steadily increased, reaching $\sim 80 \% 7$ months after transplantation, while the CD45.2 $2^{+}$cell population in the $\mathrm{PB}$ of mice receiving WT BM cells remained $\sim 50 \%$ (Fig. 3f). Interestingly, mice receiving HoxBlincTg BM cells became moribund or died 2.5-7 months after transplantation (Fig. 3g). These HoxBlincTg BM recipients displayed similar hematological phenotypes as the primary HoxBlincTg mice, including high WBC counts, dramatically elevated immature myeloid cells and neutrophils, severe anemia, and decreased platelet counts (Supplementary Fig. 5a). Flow cytometric analyses of the donor $\left(\mathrm{CD} 45.2^{+}\right)$and competitor $\left(\mathrm{CD} 45.1^{+}\right)$derived $\mathrm{BM}$ cells revealed significantly greater percentages of $\mathrm{c}-\mathrm{Kit}^{+}$and $\mathrm{Gr}-1^{+} / \mathrm{Mac}^{+}$cells and lower proportions of $\mathrm{CD}^{+}, \mathrm{B}_{22} 0^{+}$and Ter119+ cells in HoxBlincTg vs. WT recipients (Supplementary Fig. 5b, c). Strikingly, the BM CD45.2 $2^{+} \mathrm{Lin}^{-}$cells of HoxBlincTg recipients are comprised of significantly higher LK and LSK cells than that of CD45.1 ${ }^{+} \mathrm{Lin}^{-}$ cells in HoxBlincTg recipients and $\mathrm{Lin}^{-}$cells in WT recipients (Supplementary Fig. 5b). Consistently, a higher proportion of immature myeloid cells were observed in the BM of HoxBlinc Tg recipients (Supplementary Fig. 5d, e). The similar phenotypes displayed in recipients transplanted with HoxBlincTg BM cells and primary HoxBlincTg mice indicate that the aberrant HSPC function and AML-like disease induced by HoxBlinc overexpression in mice are transferable and HSPC cell-autonomous. Collectively, transgenic expression of HoxBlinc in mice enhances HSC self-renewal and expands myelopoiesis, leading to AML-like disease, reminiscent of the phenotypes seen in the $N P M 1^{\mathrm{c} /+}$ mice.

Overexpression of $\mathrm{HoxBlinc}$ activates $\mathrm{NPM}^{+} \mathrm{c}^{+}$signature genes via increased enhancer/promoter chromatin accessibility in HSPCs. To further delineate whether HOXBLINC is a downstream

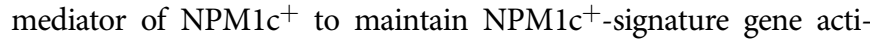
vation in HSPCs, RNA-seq was performed with WT and HoxBlincTg LSK cells from 8-week old mice. Comparison of gene expression profiles of HoxBlincTg LSK cells to those of WT LSK cells identified 1,281 differentially expressed genes $(P<0.05$ and fold-change $\geq 2.0$, Fig. 4a). Among the DEGs, 718 were upregulated and 563 were down-regulated. Strikingly, $27.3 \%$ of the upregulated genes and $21 \%$ of the down-regulated genes were overlapped with the up- and down-regulated genes in $N P M 1^{\mathrm{c} /+}$ v.s. WT LSK cells, respectively (Fig. 4b). These common up-regulated genes of HoxBlincTg and NPM1 ${ }^{\mathrm{c} /+}$ LSK cells (Supplementary Table 2) included the NPM1c ${ }^{+}$signature genes HoxB2-5, HoxA9-10, Meis1, and Runx1, which were confirmed by RT-qPCR (Supplementary Fig. 6a). Consistently, GO and GSEA analyses of the differentially expressed genes in HoxBlincTg vs. WT LSK cells revealed association/enrichment of similar transcription signatures and pathways with those in $N P M 1^{\mathrm{cl}+}$ vs. WT LSK cells, including NPM1-mutated 
a

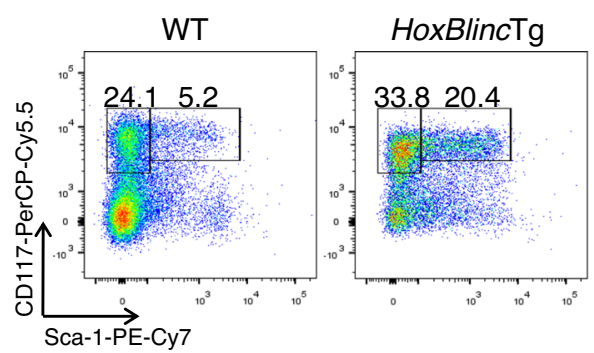

b

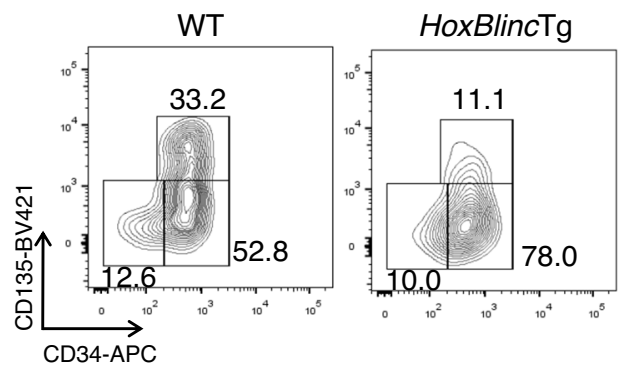

C

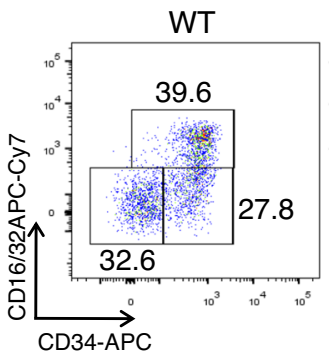

d

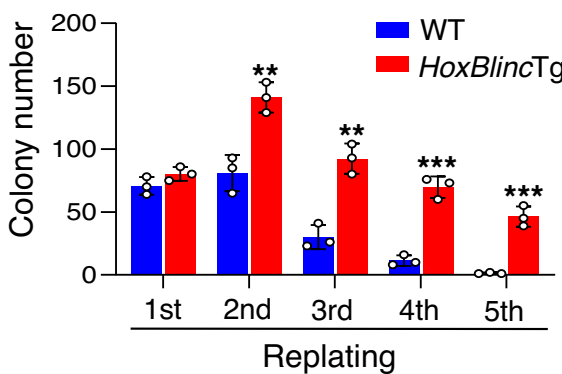

f

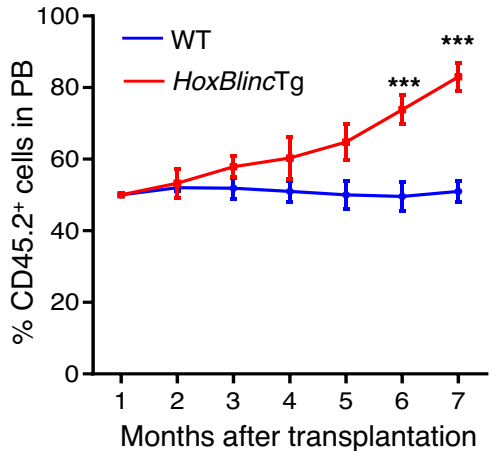

signature, HOXA9 oncogenic pathway, HSC proliferation, cell fate commitment, myeloid differentiation, Wnt and JAK-STAT signaling pathways (Fig. 4c, d, Supplementary Fig. 6b).

Since HoxBlinc promotes the expression of anterior HoxB genes by recruiting SETD1A and MLL1 complexes and
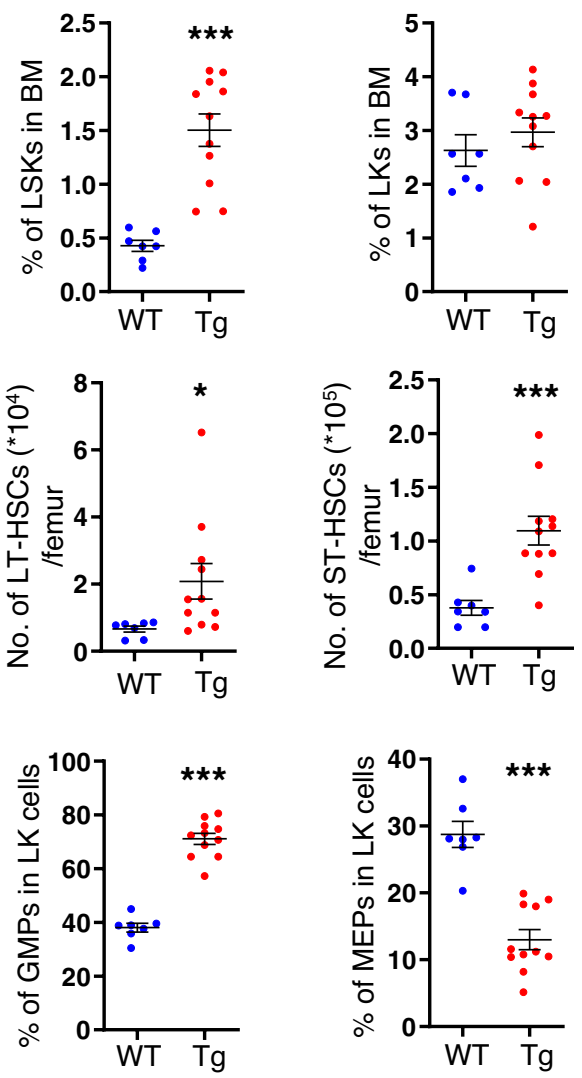

e

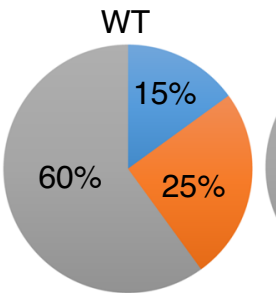

HoxBlincTg

Symmetric self renewal

Asymmetric self renewal

Symmetric differentiation

g

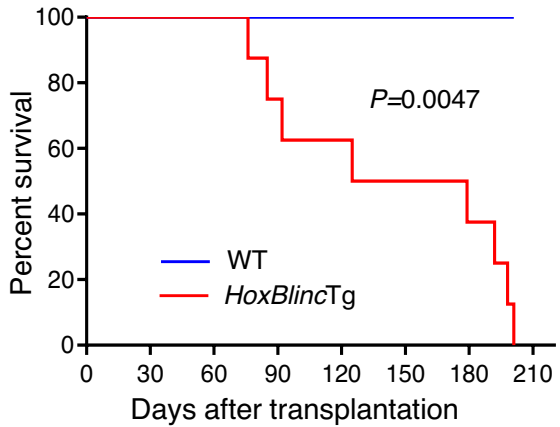

organizing active chromatin domain in the anterior HoxB locus ${ }^{10}$, HoxBlinc upregulation caused by either NPM1c ${ }^{+}$or HoxBlincTg could activate its target genes in HSPCs by accentuating enhancer/promoter chromatin accessibility. To test this, we carried out an assay for Transposase-Accessible 
Fig. 3 Transgenic expression of HoxBlinc enhances HSC self-renewal and expands myelopoiesis. a FACS analysis of LSK (Lin-SCa- $\left.1^{+}{ }^{C}-K_{i t}{ }^{+}\right)$and $L K$ ( $\mathrm{Lin}^{-} \mathrm{Sca}-1^{-} \mathrm{c}^{-} \mathrm{Kit}^{+}$) cell populations in the BM Lin ${ }^{-}$cells of representative young (8-10 weeks old) WT and HoxBlincTg mice (left). Quantitation of the percent LSK and LK cells in the total BM cells of each genotype of mice is shown (right). b FACS analysis of LT-HSC, ST-HSC, and MPP cell populations in the BM LSK cells of representative young WT and HoxBlincTg mice (left). The total number of LT- and ST-HSCs per femur in WT and HoxBlincTg mice are shown (right). c FACS analysis of GMP, MEP, and CMP populations within BM LK cells of representative young WT and HoxBlincTg mice (left). Quantitation of the percent GMP and MEP cell populations in the BM LK cells of each genotype of mice is shown (right). (Data in (a-c) are presented as mean \pm SEM; WT group $n=7$; HoxBlincTg group $n=11 ;{ }^{\star} P<0.05$ and ${ }^{\star \star \star} P<0.001$ by two-tailed unpaired Student's t-test.) $\mathbf{d}$ The number of colonies per 100 WT or HoxBlincTg BM LSK cells are shown (1st). Colonies were replated every 7 days for 4 times (2nd-5th). (bars represent mean \pm SD; $n=3$ taken from three independent experiments; two-tailed t-test; $\left.{ }^{\star \star} P<0.01,{ }^{\star \star \star} P<0.001\right)$. e Paired-daughter cell assays were performed on CD34-LSK cells clonesorted from BM cells of WT and HoxBlincTg mice, and each cell was analyzed for symmetric self-renewal (blue), asymmetric cell division (orange), or symmetric differentiation (gray). f Kinetic flow cytometric analyses of CD45.2 chimerism in the PB of recipients transplanted with WT or HoxBlincTg BM cells. (bars represent mean \pm SD; WT group $n=5$; HoxBlincTg group $n=8$; two-tailed t-test; ${ }^{\star \star \star} P<0.001$ ). $\mathbf{g}$ Kaplan-Meier survival curve of recipient mice (8 mice/genotype) transplanted with WT or HoxBlincTg BM cells together with WT CD45.1 BMs ( $P$ value was calculated by Log-rank test).

Chromatin with high throughput sequencing (ATAC-seq) using LSK cells from WT, NPM1 $1^{\mathrm{c}+}$, and HoxBlincTg mice (Supplementary Fig. 6c, d). Coincidently, NPM1 $1^{\mathrm{c} /+}$ and HoxBlincTg LSK cells shared significant portions of genes exhibiting gain $(28.9 \%)$ or loss $(24.3 \%)$ of promoter accessibility (Fig. 4e, Supplementary Table 3). In addition, significant portions of the genes with promoter accessibility gain by either $\mathrm{NPMlc}^{+}$or HoxBlinc Tg were also upregulated (Supplementary Fig. 6e). These genes included the NPM1c-signature genes HoxB2-5, HoxA9-10, Meis1, and Runxl, as well as other target genes such as Stat1 and Cdr2 (Supplementary Figs. 1d, 6f, 6g; Fig. 4f, g) that also play critical roles in HSC regulation and leukemogenesis. As a control, no significant changes in chromatin accessibility was observed in the Lypla1 locus, and the expression of Lypla1 was not altered by HoxBlinc upregulation caused by either NPM1c ${ }^{+}$or HoxBlinc Tg (Supplementary Fig. 6g). These results suggest that overexpression of HoxBlinc lncRNA specifically activates $\mathrm{NPMlc}^{+}$signature genes via enhancing enhancer/promoter chromatin accessibility in HSPCs.

HoxBlinc directly binds to target genes and mediates chromatin interactions to drive gene regulatory networks in HSPCs. CTCF boundaries facilitate enhancer/promoter interactions within confined topologically associated domains (TADs). We recently reported that a CTCF boundary in the posterior HOXA locus establishes and maintains an active TAD to drive posterior HOXA gene expression ${ }^{15}$. To examine whether HoxBlinc overexpression affects CTCF defined anterior TAD domain and enhancer/promoter regulatory networks in the anterior HoxB locus, circular chromosome conformation capture using high throughput sequencing (4C-seq) was performed using the several HoxB locus CTCF binding sites (CBSs) as viewpoints in HoxBlincTg vs. WT Lin ${ }^{-} \mathrm{c}-\mathrm{Kit}^{+}$cells (Fig. 5a). When the CBS located between HoxB4 and B5 (CBS4/5, which overlaps HoxBlinc gene) was used as a viewpoint, CBS4/5 interacted with the $+43 \mathrm{~Kb}$ CBS $(+43 \mathrm{CBS}$, Fig. 5b). CBS4/5 also contacted each of the anterior HoxB genes (Fig. $5 \mathrm{~b}$ ), suggesting that either CBS4/5 or more likely HoxBlinc communicates with anterior HoxB gene promoters. When +43 CBS was used as a viewpoint, the interaction of CBS4/ 5 and +43 CBS was confirmed and +43 CBS communicated with anterior HoxB genes too (Fig. 5b). Interestingly, HoxBlinc overexpression intensified each of these long-range interactions within the anterior $\operatorname{HoxB}$ locus mediated by CBS4/5 and/or $+43 \mathrm{CBS}$ (Fig. 5b). In contrast, $+73 \mathrm{~Kb}$ CBS $(+73 \mathrm{CBS})$ and HoxB13 CBS (CBS13) did not interact with the anterior HoxB genes, although +73CBS interacted with CBS5/6 and CBS8/9, which however was not affected by HoxBlinc overexpression (Fig. 5b). Furthermore, HoxBlinc overexpression also induced the long-range interactions of CBS4/5 and/or +43CBS with the promoter regions of HoxBlinc target genes such as Stat1, Crd2, and posterior HoxA genes, but not non-HoxBlinc target HoxD genes (Fig. $5 c$, Supplementary Fig. 7a). These data indicate that HoxBlinc coordinated with the CBS $4 / 5$ and +43 CBS in HSPCs to facilitate and maintain longrange chromatin interactions with $\mathrm{NPM}_{1}{ }^{+}$signature gene loci for their activation.

To completely understand the mechanism by which HoxBlinc overexpression regulates hematopoietic transcription program in HSPCs, we carried out ChIRP-seq (Chromatin Isolation by RNA Purification combined with deep sequencing) to map the genomic HoxBlinc binding sites in WT and HoxBlinc Tg Lin ${ }^{-} \mathrm{c}-\mathrm{Kit}^{+}$cells. Overexpression of HoxBlinc significantly increased its binding to the promoter regions of anterior $\operatorname{HoxB}$ genes and other trans targets, such as Stat1, Cdr2, Wnt5a, Runx1, and posterior HoxA genes (Fig. 5d, Supplementary Fig. 7b). The HoxBlinc binding to anterior HoxB and Runx1 promoters were confirmed by ChIRPqPCR (Supplementary Fig. 7c, d). The global HoxBlinc-binding site distribution in $\mathrm{Lin}^{-} \mathrm{c}-\mathrm{Kit}^{+}$cell genome revealed that HoxBlinc mainly interacted with noncoding regions, including intergenic regions, introns, and promoters. Emphatically, HoxBlinc overexpression markedly increased its occupancy with promoters and UTRs (Fig. 5e). Furthermore, GO analysis of HoxBlinc-bound genes found enrichment of pathways important for HSPC regulation and leukemogenesis such as Hox genes, AML, HSC proliferation, Wnt signaling, and cell cycle (Supplementary Fig. 7e). Integration of ChIRP-seq, RNA-seq, and ATAC-seq datasets from WT and HoxBlincTg HSPCs revealed that around $74 \%$ of the genes with increased HoxBlinc binding exhibited $\mathrm{a} \geq 2$ folds increase in gene expression levels (Fig. 5f) and $44.7 \%$ of them showed increased promoter chromatin accessibility (Fig. 5g). These data revealed that HoxBlinc acts as an epigenetic regulator to control target gene expression through remodeling promoter chromatin accessibility. Transcription motif analysis showed that the top HoxBlinc bound motifs in HoxBlincTg HSPCs are transcription factors important for hematopoiesis, such as CTCF and PU.1 (Supplementary Fig. 7f, Supplementary Table 4). These data demonstrated that HoxBlinc directly binds to hematopoietic specific target genes, mainly the $\mathrm{NPMlc}^{+}$signature genes, and mediates long-range chromatin interactions to drive gene regulatory networks in HSPCs.

Recruitment of MLL1 is critical for HoxBlinc overexpression mediated target gene expression and leukemogenesis. As HoxBlinc recruits SETD1A and MLL1 complexes to organize active chromatin domain in the HoxB loci in mouse ECSs derived primitive erythroid progenitor cells ${ }^{10}$, we performed RIP-qPCR analysis which showed much greater HOXBLINC enrichment in the immunoprecipitates of anti-MLL1 and anti-SETD1A, but not control IgG and anti-LSD1 antibodies in high HoxBlinc expressing OCI-AML3 cells as compared to the low HoxBlinc expressing OCI-AML2 cells (Supplementary Fig. 8a), confirming the interaction of human HOXBLINC with MLL1 and SETD1A. We next 
a

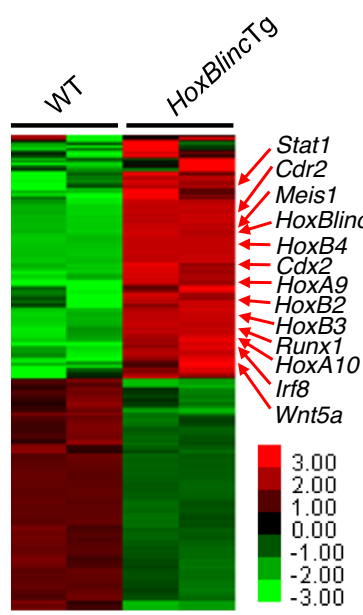

b
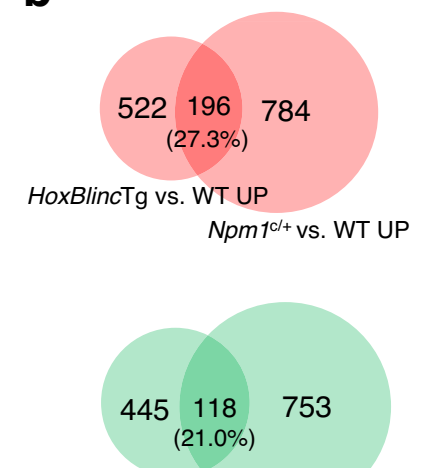

HoxBlincTg vs. WT DOWN

$N p m 1^{\mathrm{c} / \mathrm{v}}$ vs. WT DOWN
C

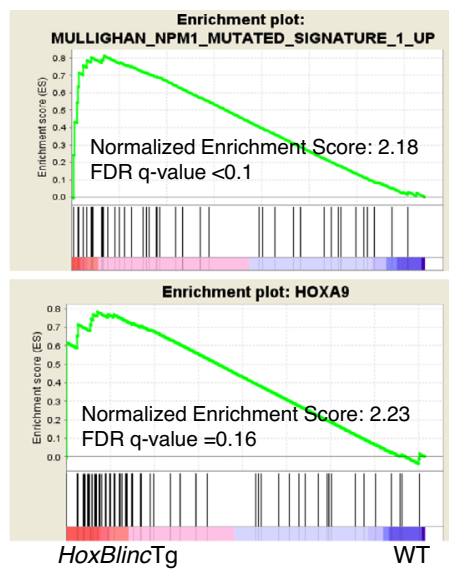

d

Hematopoietic cell lineage Cell fate commitment Endothelium development Post-embryonic development

Hematopoietic stem cell proliferation Myeloid differentiation Mesodermal cell differentiation Stem cell differentiation

PI3K-Akt signaling pathway BMP signaling pathway Wnt signaling Pathway Jak-STAT signaling Pathway

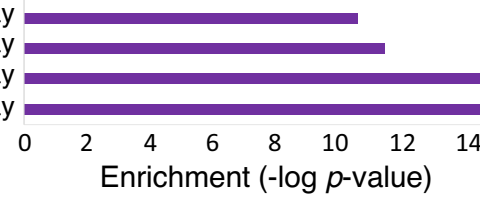

HoxBlincTg vs. WT LSK cells e

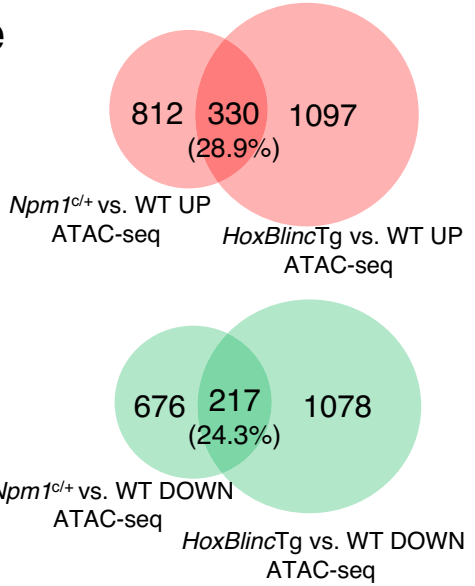

f

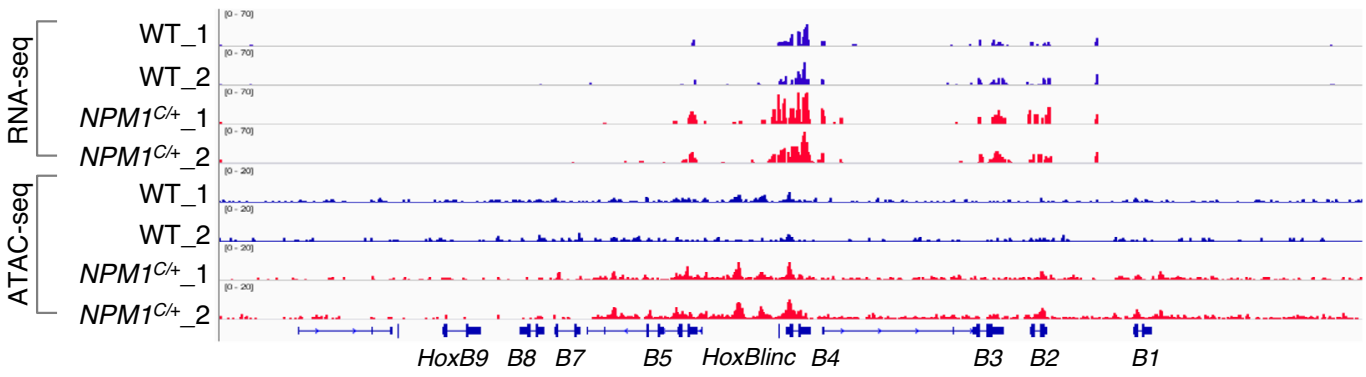

g

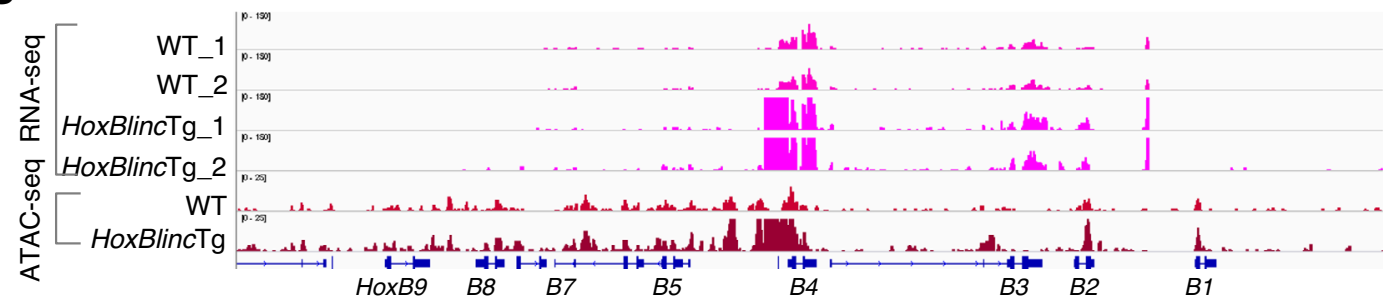

Fig. 4 Overexpression of HoxBlinc activates NPM1c ${ }^{+}$signature genes via enhancing promoter chromatin accessibility in LSK cells. a Heatmap of RNAseq analysis shows the up- and down-regulated genes in HoxBlincTg vs. WT LSK cells based on two independent experiments. Red arrows: up-regulated genes implicated in HSPC regulation/leukemogenesis. b Overlap of up- (top) or down- (bottom) regulated genes between HoxBlincTg vs. WT and NPM7c/+ vs. WT LSK cells. c Enrichment of upregulated genes involved in NPM1-mutated signature (top) and HOXA9 (Bottom) oncogenic pathway upon overexpression of HoxBlinc in LSK cells by GSEA. d The HoxBlinc overexpression dysregulated genes in LSK cells were analyzed and annotated by GO analysis. e Overlap of global gain (top) or loss (bottom) of promoter chromatin accessibilities between ATAC-seq data of HoxBlincTg vs. WT and NPM7c/+ vs. WT LSK cells. f RNA-seq (chromatin accessibility, top 4 tracks) and ATAC-seq (gene expression, bottom 4 tracks) analysis of WT and Npmic/+ LSK cells in the HoxB gene locus. $g$ RNA-seq analysis (top 4 tracks) and ATAC-seq analysis (bottom 2 tracks) of WT and HoxBlincTg LSK cells in the HoxB gene locus. 

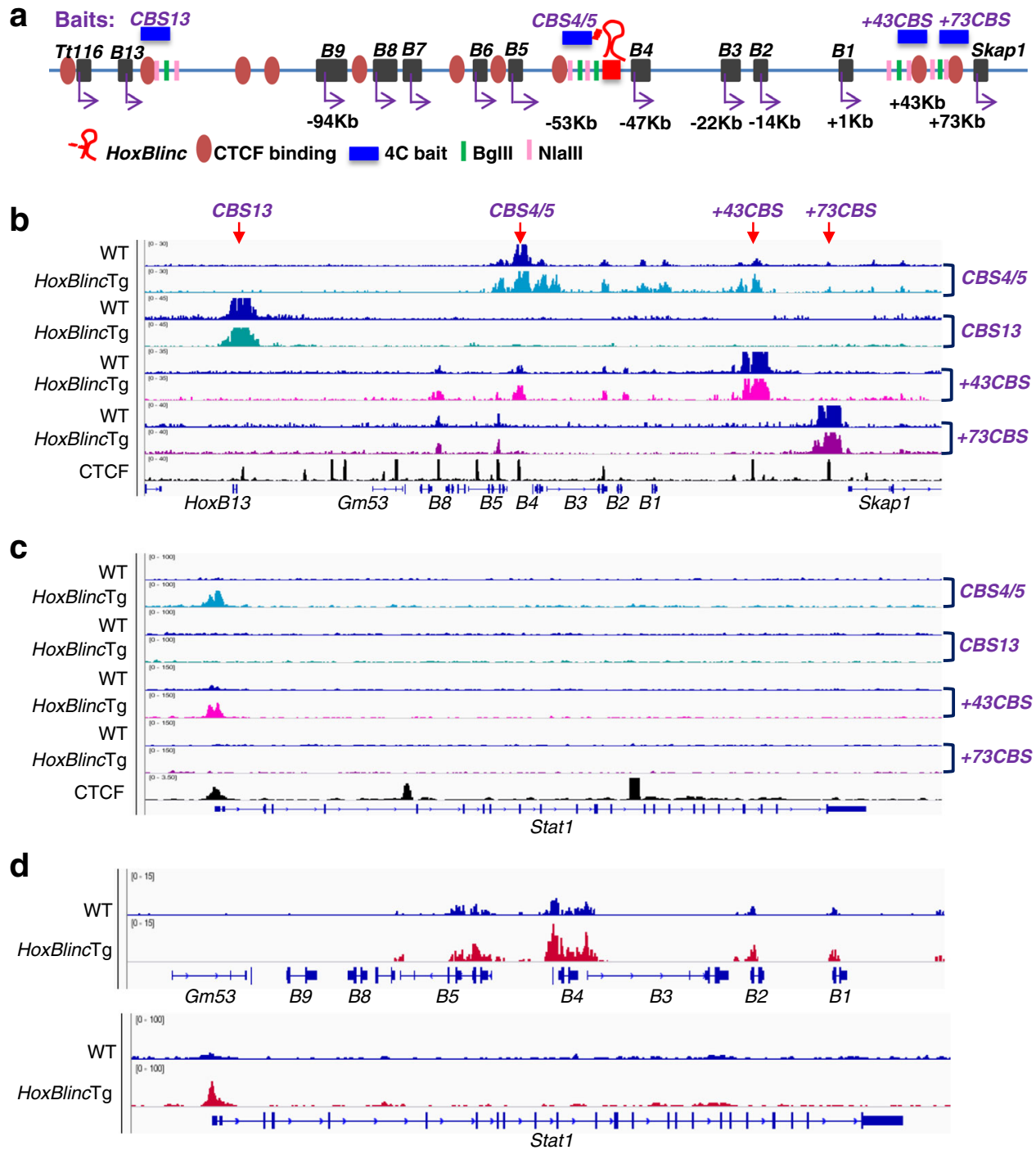

e

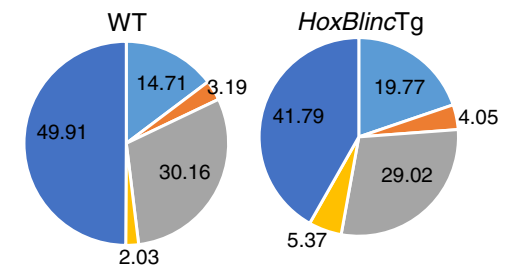

- Promoter $=$ Exon $=$ Intron $\approx$ UTR $=$ Intergenic

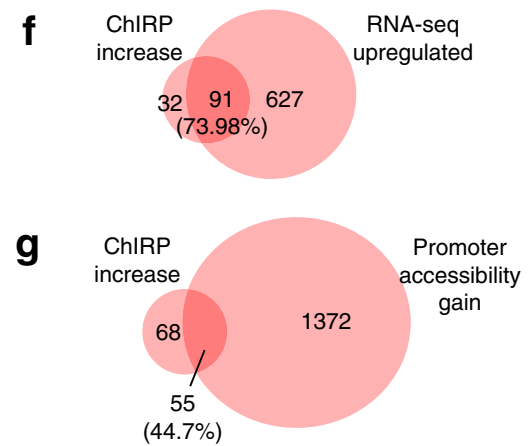

Fig. 5 HoxBlinc directly binds to target genes and mediates chromatin interactions to drive gene regulatory networks in HSPCs. a Schematic diagram showing the CTCF binding sites (CBS), location of the 4 C baits in HoxB locus of the mouse genome. $\mathbf{b}$, $\mathbf{c}$ Long-range chromatin interactions with HoxB locus (4 baits) as determined by 4C-seq analysis in WT and HoxBlincTg Lin ${ }^{-} \mathrm{C}^{-} \mathrm{Kit}^{+}$cells. HoxBlinc overexpression increased the interactions of CBS4/5 and +43 CBS with the HoxB genes (b), HoxBlinc overexpression also enhanced the interaction of CBS4/5 or $+43 C B S$ with Stat1 promoter region (c), ChIP-sea analysis of CTCF binding sites was obtained from the NCBI GEO public database (GSM918748). d ChIRP-seq analysis of HoxBlinc bindings to the HoxB and Stat1 gene loci in WT and HoxBlinc Tg Lin ${ }^{-}{ }^{-}-K_{i t}{ }^{+}$cells. e The pie chart shows the distribution of promoter, exon, intron, UTR, and intergenic region within the total HoxBlinc binding sites in WT (left) and HoxBlincTg (right) Lin ${ }^{-} \mathrm{c}-\mathrm{Kit}^{+}$cells as identified by ChIRP-seq. f Overlap of genes with HoxBlinc binding gain to their promoter regions as identified by ChIRP-seq and the upregulated genes as identified by RNA-seq in HoxBlincTg vs. WT HSPCs. $\mathbf{g}$ Overlap of genes with HoxBlinc binding gain to their promoter regions as identified by ChIRP-seq and genes with promoter accessibility gain as identified by ATAC-seq in HoxBlincTg vs. WT HSPCs. 
examined whether SETD1A and MLL1 are important for HoxBlinc overexpression mediated abnormal HSPC function and leukemogenesis both in vitro and in vivo. However, knockdown Mll1, but not Setd1a in HoxBlincTg LSK cells was capable of mitigating the abnormal replating potential mediated by HoxBlinc overexpression (Fig. 6a, Supplementary Fig. 8b). Furthermore, the genetic deletion of one Setd1a allele in HoxBlincTg mice (Setd1 $a^{+/-} ;$HoxBlincTg) did not rescue the abnormal hematologic phenotypes induced by HoxBlinc overexpression in vivo (Supplementary Fig. 8c-f), indicating that SETD1A is unlikely to be a key player in HoxBlinc overexpression mediated abnormal hematopoiesis. When transplantation was performed using HoxBlincTg $\mathrm{Lin}^{-} \mathrm{c}-\mathrm{Kit}^{+}$cells transduced with lentivirus expressing scramble control or shMll1, Mll1 KD significantly prolonged the survival of recipients receiving shMll1-expressing HoxBlincTg $\mathrm{Lin}^{-} \mathrm{c}-\mathrm{Kit}^{+}$cells as compared to shScramble expressing HoxBlincTg Lin $^{-} \mathrm{c}-\mathrm{Kit}^{+}$cells (Fig. 6b). And the aberrant expansion of $\mathrm{CD}_{11} 7^{+} / \mathrm{CD} 11 \mathrm{~b}^{+}$immature myeloid cells and GMPs, as well as anemia in HoxBlincTg mice, were also largely restored by Mll1 KD (Fig. 6c, d). These data indicate that Mll1 KD is capable of mitigating the AML development induced by HoxBlinc overexpression.

Since MLL1 is critical for HoxBlinc overexpression mediated abnormal hematopoiesis, HoxBlinc overexpression in HSPCs might activate its target genes by increasing MLL1 recruitment and thereby enhancing $\mathrm{H} 3 \mathrm{~K} 4 \mathrm{me} 3$ occupancy to facilitate enhancer/promoter chromatin accessibility. To confirm this, we carried out MLL1 and H3K4me3 ChIP-seq using WT and HoxBlincTg LSK cells. Combined ChIP-seq and CHIRP-seq analyses revealed significantly high overlap for the genomic binding sites of HoxBlinc and MLL1 (Fig. 6e-g, Supplementary Fig. 9a). Impressively, $51.2 \%$ of the genes with increased HoxBlinc binding exhibited elevated MLL1 recruitment and most of which (31.7\%) also showed increased H3K4me3 occupancy, including the $\mathrm{NPM}_{1}{ }^{+}$-signature genes such as anterior $\mathrm{HoxB}$, posterior HoxA, Meis1, and Runx1, as well as other hematopoietic genes such as Stat1 and Cdr2 (Fig. 6e-g, Supplementary Fig. 9a, Supplementary Table 5). In contrast, no significant changes in MLL1 recruitment and $\mathrm{H} 3 \mathrm{~K} 4 \mathrm{me} 3$ occupancy were observed in the control Lypla1 (no change in HoxBlinc binding), Eno1 (no HoxBlinc binding), and Car6 (no HoxBlinc/MLL1 binding) loci (Supplementary Fig. 9b); and the expression of such genes was not altered by HoxBlinc overexpression. These data demonstrate that HoxBlinc overexpression mediates target gene expression via increased recruitment of MLL1 and subsequent enhancement of H3K4me3 occupancy.

\section{Discussion}

In a humanized $N P M 1 c^{+}$knock-in mouse model, NPM1c ${ }^{+}$ enhanced HSC self-renewal and expanded myelopoiesis leading to $1 / 3^{\text {rd }}$ of the animals developing late-onset $\mathrm{AML}^{14}$. Brunetti et al. recently reported that specific reduction of NPM1c ${ }^{+}$lessens key features of the leukemic program ${ }^{16}$. Thus, NPM1 mutation is an AML-driving lesion and maintains leukemia mainly through a gainof-function by the $\mathrm{NPM}^{+} \mathrm{c}^{+}$. $\mathrm{NPM}^{+} \mathrm{c}^{+}$-mediated leukemogenesis has been shown to depend on the unique gene expression signatures such as $H O X A / B$ and MEIS1 activation ${ }^{17}$. However, how this aberrant gene expression program is driven and maintained is largely unknown. In this study, we show that NPM1c ${ }^{+}$deregulates its signature genes, perturbs hematopoiesis, and promotes leukemogenesis via the activation of a critical lncRNA, HOXBLINC. HOXBLINC overexpression is strongly associated with NPM1 mutations in AML and NPM1c ${ }^{+}$expression leads to HoxBlinc activation in HSPCs. Although HoxBlinc is involved in normal hematopoietic development ${ }^{13}$, HoxBlinc overexpression plays an essential and sufficient oncogenic role in NPM1c ${ }^{+}$-mediated signature gene expression, HSPC deregulation, and leukemogenesis. Therefore, our studies identify HoxBlinc activation as a critical downstream mediator for NPM1c ${ }^{+}$.

In addition to mutations and/or aberrant expression in protein-coding genes, misregulation of lncRNAs perturbs cellular physiology in multiple ways and plays important roles in the development and progression of various cancers ${ }^{18}$. However, how lncRNAs affect the initiation and progression of malignant myelopoiesis remains to be determined. Accordingly, lncRNAs have been profiled in various myeloid leukemias in order to identify potential oncogenic lncRNAs ${ }^{19-21}$. Recent studies have shown that LncHSC-2 and Hottip lncRNAs contribute to the control of critical signaling pathways in HSC regulation ${ }^{12,22}$. However, a direct link between IncRNAs and oncogenesis remains elusive in malignant myelopoiesis. Furthermore, the detailed molecular mechanisms underlying lncRNA dysregulation-mediated myeloid malignancy development remain largely unknown. Our RNA-seq analyses on HOXBLINCi vs. control NPM1c $c^{+}$OCI-AML3 cells and HoxBlincTg vs. NPM1 ${ }^{\mathrm{c} /+}$ or WT LSK cells demonstrate that HOXBLINC regulates

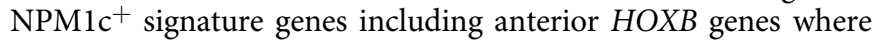
HOXBLINC resides and genes located on other chromosomes such as HOXA9-10, MEIS1, and RUNX1. ChIRP-seq analyses reveal that HoxBlinc binds to the promoters of both resident anterior $\operatorname{Hox} B$ cluster genes and distant target genes such as posterior HoxA and Runx1. These results indicate that the regulation of the NPM1c ${ }^{+}$signature genes by HoxBlinc is achieved through the direct HoxBlinc binding via cis and trans actions. Indeed, GSEA and GO analyses signify enrichment of genes for NPM1-mutated signature, HOXA9 oncogenic pathway, Wnt and JAK-STAT signaling pathways in HOXBLINCi vs. control OCIAML3 cells and HoxBlincTg or $N P M 1^{\mathrm{c} /+}$ vs. WT LSK cells. Additional functional analyses demonstrate that normal hematopoiesis requires a tightly controlled HOXBLINC expression, and its misregulation caused by $\mathrm{NPM}^{+} \mathrm{c}^{+}$is an oncogenic event in leukemogenesis. Our findings on the direct oncogenic role of HOXBLINC in NPM1-mutated AML could serve as a blueprint for implicating lncRNAs in AML leukemogenesis.

LncRNAs show a high versatility in their mechanism-of-action, influencing many cellular processes such as spatial conformation of chromosomes, chromatin modifications, and RNA transcription ${ }^{23}$. HoxBlinc recruits MLL1 and SETD1a to anterior HoxB loci and controls their gene expression by regulating chromatin states during development ${ }^{10}$. Recently, SETD1A was demonstrated to act as a positive epigenetic regulator of HSC function during hematopoiesis ${ }^{24,25}$. However, genetic deletion of one Setd1a allele could not rescue HoxBlinc overexpression mediated abnormal HSPC function and leukemogenesis. On the other hand, our Mll1 $\mathrm{KD}$ experiments revealed that loss of MLL1 mitigates HoxBlinc overexpression induced abnormal HSPC function and leukemogenesis in vivo, demonstrating MLL1 as a dependency in $\mathrm{NPMlc}^{+} /$ HoxBlinc-overexpressing AML. We further showed that HoxBlinc overexpression increases HoxBlinc occupancy (in cis and trans actions) and MLL1 recruitment at the promoters of its target genes including the $\mathrm{NPM}^{+} \mathrm{c}^{+}$signature genes to induce aberrant longrange chromatin interaction networks and promote their expression. These results are in line with a recent study by Kuhn et al. showing that the chromatin binding of MLL1 is critical for NPM1mutated leukemias and Menin-Mll1 interaction controls the expression of HOX, MEIS1, and FLT3 genes in NPM1-mutated $\mathrm{AML}^{17}$. Interestingly, NPM1 mutations are mutually exclusive with several genetic abnormalities such as the $M L L$-rearrangement and MLL1-partial tandem duplication in $\mathrm{AML}^{26,27}$. Thus, in NPM1mutated AML, NPM1c ${ }^{+}$achieves and maintains its signature gene expression program via HoxBlinc overexpression, which increases 

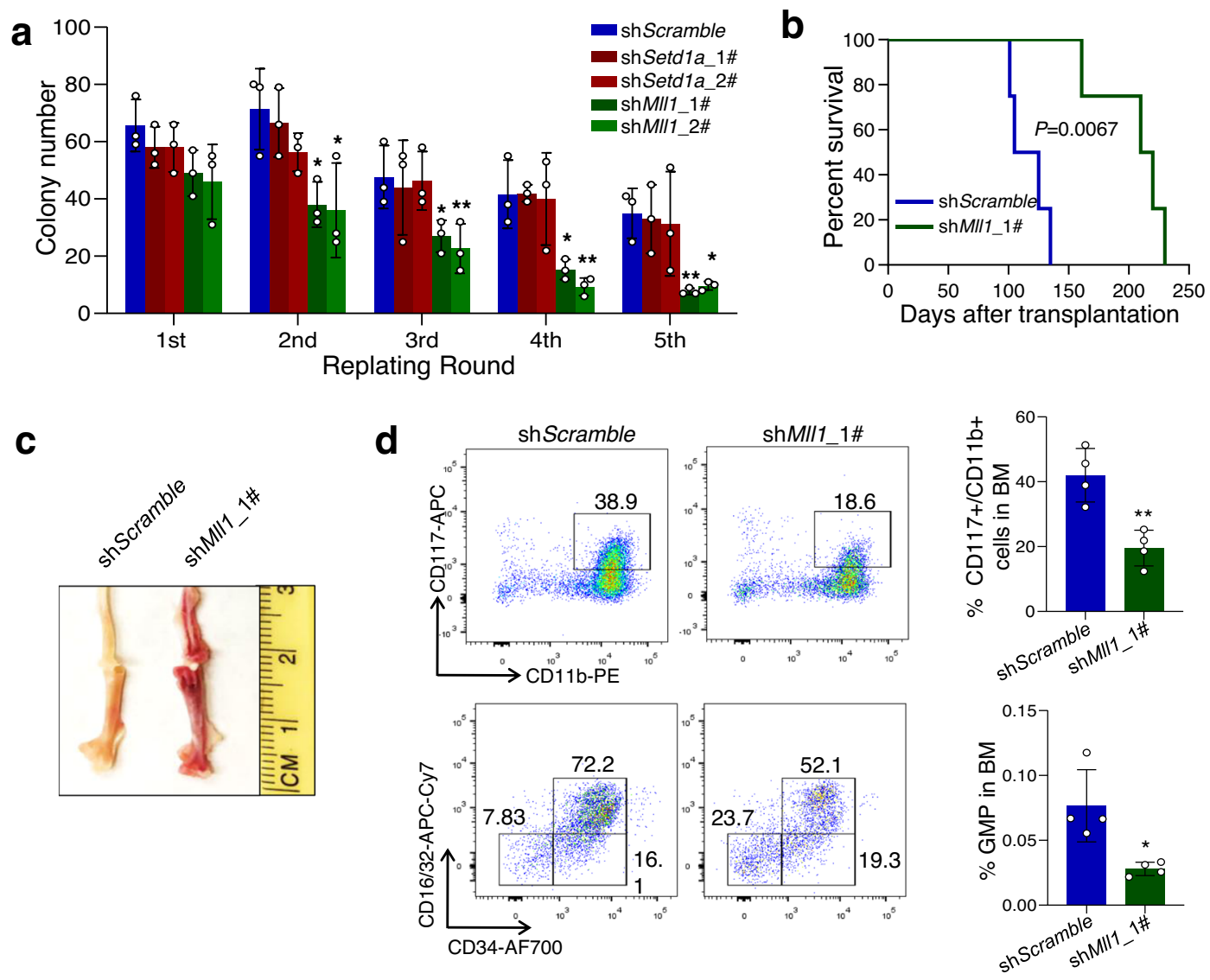

e

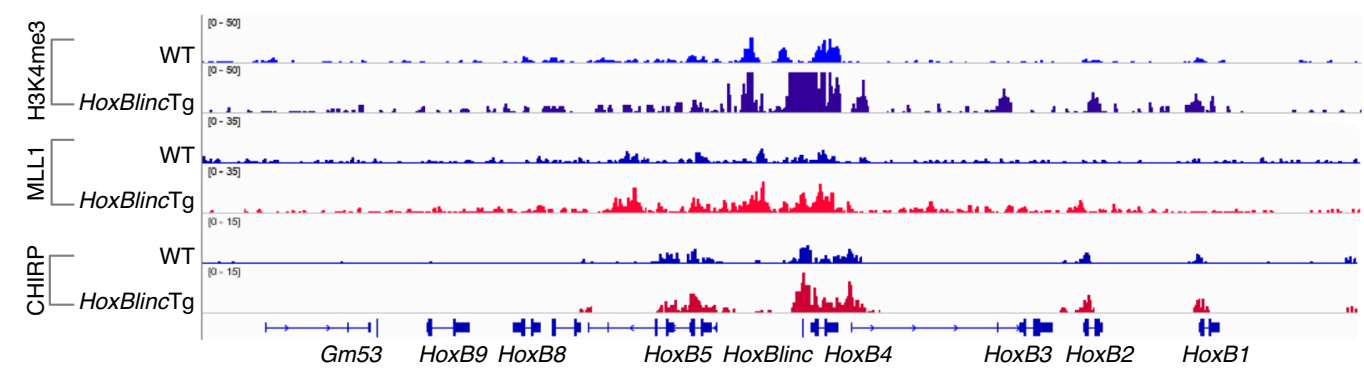

f
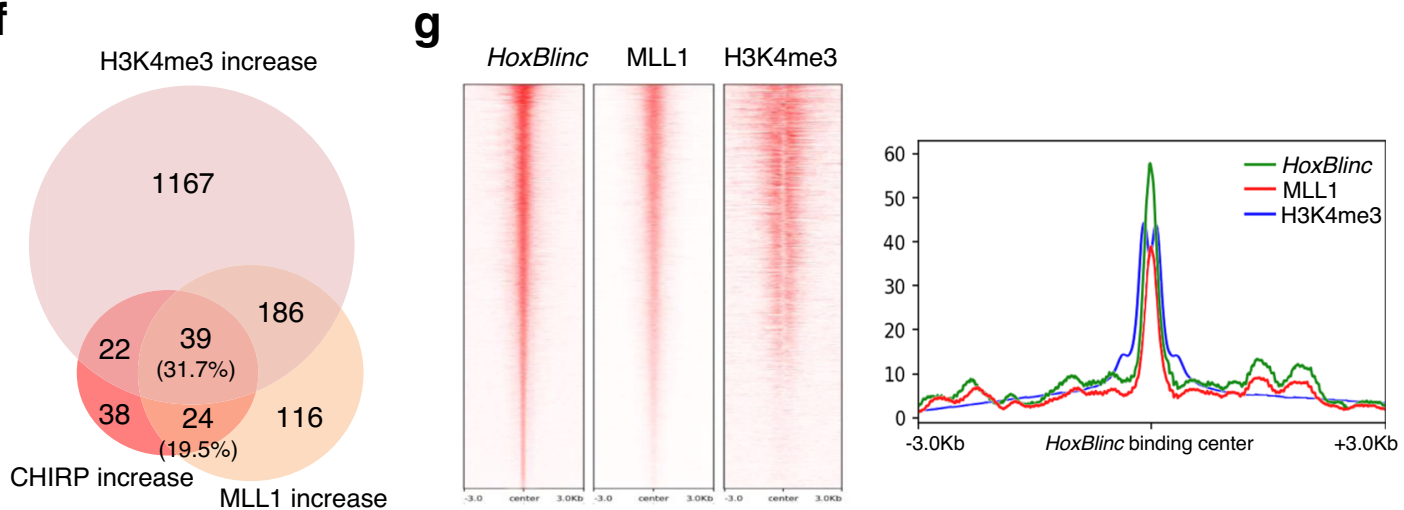

MLL1 recruitment and induces aberrant chromatin structure at $\mathrm{NPM}^{+} \mathrm{c}^{+}$signature genes.

LncRNAs are promising candidates for targeted cancer therapy, especially when they act as tissue-specific drivers of cancer. However, to the best of our knowledge, there are no therapeutic examples targeting lncRNAs in AML to date. HOXBLINC is highly expressed in HSCs. Given the profound impacts of HOXBLINC overexpression on promoting HSC self-renewal and AML initiation, targeted therapeutics could be developed to suppress the overexpressed HOXBLINC IncRNA in NPM1-mutated AML. To 
Fig. 6 Recruitment of MLL1 is critical for HoxBlinc overexpression mediated target gene expression and abnormal HSPC function. a Number of colonies per 100 sorted GFP ${ }^{+}$HoxBlincTg BM LSK cells transduced with ShScramble, shSetd1a, or shMII1 lentivirus are shown (1st). Colonies were replated every 7 days for 4 times (2nd-5th). (Data from 3 independent experiments are presented as mean \pm SD; ${ }^{\star} P<0.05$ and ${ }^{\star *} P<0.01$ by two-tailed unpaired Student's t-test.) b Kaplan-Meier survival curve of recipient mice receiving ShScramble or shMII1 LSK cells (Log-rank test was used for statistic analysis, $n=$ 4 mice/group). c Gross appearance of femur dissected from mice transplanted with shScramble or shMII1 LSK cells. d FACS analysis of BM CD11b+/ CD117+ (top) and GMP (bottom) cell populations from recipient mice (4 mice/group) transplanted with shScramble or shMII1 LSK cells. Data are presented as mean $\pm \mathrm{SD}$; ${ }^{\star} P<0.05$, ${ }^{\star \star} P<0.01$ by two-tailed unpaired Student's t-test. e ChIP-seq analysis of H3K4me3 (top 2 tracks) and MLL1 (middle 2 tracks), as well as ChIRP-seq analysis of HoxBlinc (bottom 2 tracks) in the HoxB loci of WT and HoxBlincTg Lin $^{-}{ }^{-}$-Kit $^{+}$cells. $\mathbf{f}$ The overlap among genes with gain of HoxBlinc, H3K4me3, and MLL1 bindings to their promoter regions based on ChIRP-seq and ChIP-seq analyses. g Heatmap (right) and peaks density (left) to show the overlap of HoxBlinc ChIRP-seq, MLL1, and H3K4me3 ChIP-seq.

support this, we show that knockdown of HOXBLINC, likely through the normalization of the aberrant $\mathrm{NPM}^{+} \mathrm{c}^{+}$signature gene expressions, prolongs the survival of mice transplanted with NPM1c ${ }^{+}$AML cells. In line with our findings, Qian et al. recently reported that CRISPR-Cas9-mediated specific DNA methylation at DERARE attenuates $H O X B$ gene expression and alleviates leukemogenesis ${ }^{28}$.

Our studies have provided convincing evidence that NPM1c + upregulates a set of signature gene expression including RUNX1 gene via an increased HOXBLINC expression. However, an important question remains, which is how HOXBLINC is upregulated in NPM1c + AML. Understanding of the underlying mechanisms by which HOXBLINC itself is regulated in AML could provide insight into NPM1c + -driven pathogenesis, but also lead to a strategy for the treatment of NPM1c + AML via blocking HOXBLINC activation. Although RUNX1 and NPM1 mutations are mutually exclusive in $\mathrm{AML}^{29}$, RUNX1, a critical transcription factor in HSPCs and myelopoiesis, is frequently dysregulated in AML both genetically and transcriptionally. Instead of a unidirectional NPM1c $+-H O X B L I N C$-RUNX1 axis, NPM1c may also directly activate RUNX1 expression independent of HOXBLINC leading to a positive feedback loop to control the RUNX1 and HOXBLINC expression. Another scenario could be that cytosolic relocation of NPM1c + alters CTCF-mediated AML genome topology-driven leukemic transcription networks. It has been reported that nuclear NPM1 associates with CTCF and acts as a nuclear matrix anchor for CTCF-mediated chromatin looping ${ }^{30}$. Indeed, CTCF boundaries were involved in defining transcriptionally active anterior HoxB domain including HoxBlinc lncRNA in Hox gene activation during embryonic development ${ }^{10}$. Thus, it warrants further investigation of upstream signal/transcription networks leading to aberrant homeotic transcription program in AML leukemogenesis.

In summary, we show that HOXBLINC overexpression is a critical event to drive leukemogenesis by establishing aberrant $\mathrm{NPM}^{+}{ }^{+}$signature gene expression program via controlling the MLL1 recruitment, chromatin domains, and promoter accessibility in cis and trans actions. Our studies, therefore not only provide molecular insights into the biology of HSC and NPM1mutated AML, but also create a unique opportunity for the identification of drug targets for $N P M 1 c^{+}$AML.

\section{Methods}

\section{Generation of the HoxBlinc transgenic (Tg) mouse model. All studies were} conducted in accordance with the regulatory guidelines by the Institutional Animal Care and Use Committee (IACUC) at the UT Health San Antonio and University of Miami Miller School of Medicine. The full-length of mouse HoxBlinc cDNA was cloned into HS321/45-vav vector ${ }^{31}$ by SfiI and NotI so the transgene locates between the Vav1 promoter and Vav1 enhancer. This makes the transgene specifically express in hematopoietic system. The circular plasmid was then linearized by SacII into 2 fragments, only the longer fragment (Vav1 promoter-HoxBlinc-Vav1 enhancer, $\sim 14 \mathrm{~kb}$ ) was extracted and injected into the nuclear of zygotes of C57BL/6 mice while the shorter fragment (pBlueScript II SK backbone, $\sim 3 \mathrm{~kb}$ ) was discarded. Two HoxBlinc Tg founder mice were obtained by genotyping the tail genomic DNAs with P1 (forward primer, to detect both endogenous and transgenic HoxBlinc gene) and P2 (reverse primer, to specifically recognize the transgenic HoxBlinc gene), see primer sequences in Supplementary Table 6. Transgenic mice showed a $589 \mathrm{bp}$ band while the WT mice showed no band. Both founder mice were then crossed with WT C57BL/6 mice, the HoxBlinc negative littermates were used as controls and the HoxBlincTg mice were used as experimental group throughout the study. These two lines of HoxBlincTg were analyzed separately. The mHoxBlinc-set1 and mHoxBlincset 2 were used as real-time PCR primers to recognize both endogenous and exogenous HoxBlinc lncRNA (Fig. 2d, Supplementary Table 6). The transgenic HoxBlinc expression levels were also confirmed by RNA-seq analysis (Fig. 4a).

Mouse housing conditions. A 14-hour light/10-hour dark cycle is used. Researchers do not enter the mouse room during the dark cycle. Room temperatures were set to $21^{\circ} \mathrm{C}$ with $40-60 \%$ humidity. Immunodeficient mice (NSG) were housed in the same room condition but in a separate room established for immunodeficient mice at the UT Health SA or University of Miami.

Morphological and histological analyses of the hematopoietic organs. PB was collected by tail vein bleeding and was subjected to an automated blood count (Hemavet System 950FS). PB smears were subjected to May-Grünwald-Giemsa staining for morphological and lineage differential analyses. Morphological evaluation of BM and spleen cells were performed on cytospins followed by MayGrünwald-Giemsa staining. For histopathological analyses, femurs were fixed in $10 \%$ Neutral Buffered Formalin and demineralized in a solution of $10 \%$ EDTA for 1-2 weeks, and the soft tissues such as spleen, lymph node, and liver were fixed in $10 \%$ Neutral Buffered Formalin. These fixed specimens were dehydrated with ethanol, cleared in xylenes, and then embedded in melted paraffin and allowed to harden. $5 \mu \mathrm{m}$ sections were cut and floated onto microscope slides. For routine assessment, slides were stained with hematoxylin and eosin (H\&E staining). For MPO and hCD45 immunohistochemical staining, the tissue was rehydrated followed by heat-induced epitope retrieval, peroxidase, and serum blocking. Samples were then incubated with anti-MPO (R\&D, \#MAB3174, dilution: 1:500) or antihCD45 (Abcam, ab10559, dilution: 1:1000) antibody (overnight at $4{ }^{\circ} \mathrm{C}$ followed by staining with the biotinylated secondary antibody. Slides were visualized under a Nikon TE2000-S microscope. Images were taken by a QImaging camera and QCapture-Pro software (Fryer Company Inc.). Chemicals were obtained from Sigma (St. Louis, MO) unless otherwise indicated.

Flow cytometry analysis, cell sorting, and colony assay. The peripheral blood WBCs were obtained by treating peripheral blood with red blood cell lysis buffer (QIAGEN 1045722). BM, spleen, and peripheral blood WBC cells were stained with indicated flow antibodies (flow antibodies were listed in Supplementary Table 7, lineage flow antibody was diluted as 1:25, all other flow antibodies were diluted as 1:50). The flow data was collected by BD LSRII or LSR Fortessa and analyzed with FlowJo.V10 software. The LSK cells used for colony and replating assay were purified by flow sorting: total BM cells of 6-8 weeks old mice were prepurified with lineage depletion beads (MiltenyiBiotec, Bergisch Gladbach, Germany) and then incubated with c-Kit, lineage, and Sca-1 antibodies (see Supplementary Table 7 for antibody information) and then sorted on BD FACS AriaII. IIThe purity of sorted LSK cells were routinely over 98\%. The LSKs were incubated in methylcellulose medium (Methocult M3231) which was diluted with RPMI1640, supplemented with $30 \%$ FBS, $2 \%$ BSA, and a combination of cytokines (mG-CSF, $10 \mathrm{ng} / \mathrm{mL}$; mIL-3, $5 \mathrm{ng} / \mathrm{mL}$; mEPO, $4 \mathrm{U} / \mathrm{mL}$; hTPO, $100 \mathrm{ng} / \mathrm{mL}$; and $\mathrm{mSCF}$, $100 \mathrm{ng} / \mathrm{mL}$ ) for colony assay, colony numbers were counted at 7 days. For replating assay, the colonies were passaged sequentially every 7 days for 4 times. The gating strategy of Flow analysis and sorting were included in Supplementary Table 9.

Competitive repopulation assay. A total of $1 \times 10^{6} \mathrm{BM}$ cells prepared by mixing $5 \times 10^{5}$ CD45.2 (WT or HoxBlinTg) with $5 \times 10^{5}$ CD45.1 (B6.SJL) were injected into the tail veins of lethally irradiated $(950 \mathrm{cGy})$ B6.SJL recipients (CD45.1). The contribution of CD45.1 $1^{+}$vs. CD $45.2^{+}$cells in the $\mathrm{PB}$ was monitored every month for 6 months after transplantation.

Paired-daughter cell assay. To examine the frequency of HSCs to undergo selfrenewal and differentiation, we performed paired-daughter cell assays ${ }^{32}$. Single CD34- LSK cells from BM of WT and HoxBlincTg mice were clone-sorted into 
96-well plates. The cells were maintained in RPMI1640 media supplemented with $\mathrm{mSCF}(100 \mathrm{ng} / \mathrm{mL})$ and hTPO $(50 \mathrm{ng} / \mathrm{mL})$. After the first cell division, the two daughter cells were separated, one per well for an additional 12 days in the media supplemented with mSCF, hTPO, mEPO, mIL-3, and mG-CSF. The self-renewal and differentiation capabilities of cultured CD $34^{-}$LSK cells were determined by morphological analyses of the progenies of the two daughter cells microscopically following May-Grünwald-Giemsa staining. A total of 192 single cells (two 96-well plates) were analyzed to calculate the percentage of symmetric/asymmetric cell divisions.

\section{Human AML samples and patient data analysis. All human samples from} healthy donors and patients with primary AML were obtained after informed consent following the guidelines of the Institutional Review Board of Pennsylvania State University College of Medicine (IRB protocol \#2000-186 and \#29252 EP) or the Institute of Hematology and Blood Disease Hospital, Tianjin, China. For HOXBLINC gene expression level analysis, BM low-density mononuclear cells (MNCs) were purified using Ficoll-Hypaque. RNA specimens extracted from MNCs of AML patients, MNCs of healthy controls or CD34 ${ }^{+}$cells of healthy controls were treated with RNase-free DNase to remove contaminating genomic DNA and first-strand CDNA was then synthesized. Real-time PCR was performed using Fast SYBR Green master mix. PCR amplifications were performed in triplicate with parallel measurements of human GAPDH (internal controls). The expression levels of HOXBLINC IncRNA were also assessed by analyzing a TCGA AML dataset from 179 AML patients (GEO accession number: GSE62944).

\section{RNA isolation, quantitative RT-PCR, as well as RNA-sequencing and data} analysis. Total RNAs were extracted and purified with the RNeasy mini-isolation kit according to the manufacturer's instructions (Qiagen, MD, USA). A total of $2 \mu \mathrm{g}$ RNA was subjected to reverse-transcription with Superscript II Reverse Transcriptase (Invitrogen) and analyzed by a real-time PCR Detection System (Bio-Rad). Primer sequences for qPCR are listed in Supplementary Table 6 and key reagents are listed in Supplementary Table 7. For RNA-seq library generation, RNA sequencing library was prepared with the Illumina TruSeq mRNA sample preparation system kit (Cat\# 20020594). In brief, total RNA samples were purified with purification beads, and then fragmented with the fragmentation buffer mix. Next, First strand cDNA was synthesized with the First Stand Synthesis Act D mix. Then, the second strand cDNA was synthesized with the Second Strand Marking Master Mix. After that, RNA-seq libraries were amplified and indexed with the adapter primers. Then, the quality of the library was tested with Qubit and Agilent Bioanalyzer. Final libraries were submitted to paired-end sequencing of $50 \mathrm{bp}$ length on an Illumina HiSeq 3000. For RNA-seq data analysis, cutadapt (http:// cutadapt.readthedocs.io, version 1.2.0) program was used to trim the adaptors and low quality reads from the RNA-seq raw data files ${ }^{33}$. Then, all of the filtered sequencing reads were processed and aligned to the mouse genome assembly (mm9) or human genome assembly (hg19) using TopHat (version 2.0) and Bowtie $2^{34-36}$. Next, FPKM (paired-end fragments per kilobase of exon model per million mapped reads) was calculated for each gene and further normalized. The differential expression was determined according to the FPKM value and processed with the Cufflinks v2.2.1 and Cuffdiff ${ }^{37}$. Next, differentially expressed genes with greater than 2.0-fold were identified through comparing WT control and experimental groups according to the FPKM values. The scatter plot was generated according to the $\log _{2}$ transformation of the FPKM values, and the upregulated or downregulated genes with more than two folds changes were marked with blue dots, and no change genes were marked with red dots. Gene Ontology analysis was carried out with the Database for Annotation, Visualization and Integrated Discovery (DAVID) tool (https://david.ncifcrf.gov/, Version 6.8) ${ }^{38}$. Gene set enrichment analysis (GSEA) was conducted according to recommended parameters (http://software.broadinstitute.org/gsea/doc/GSEAUserGuideFrame.html) using gene sets obtained from the Molecular Signatures Database ${ }^{39}$. The normalized expression data tracks were loaded to the Integrated Genomic Viewer (IGV) for visualization. The sequence reads have been deposited in the NCBI GEO dataset (GSE115096). Key software and algorithms used are listed in Supplementary Table 7.

RNA immunoprecipitation (RIP) assay. The RNA-IP protocol was performed according to the previous reports ${ }^{40,41}$. In brief, OCI-AML3 and OCI-AML2 cells were harvested and washed with PBS, centrifuged and re-suspended in freshly RIP nuclear isolation buffer (1.28 M sucrose, $40 \mathrm{mM}$ Tris-HCl pH 7.5, $20 \mathrm{mM} \mathrm{MgCl}_{2}$, $4 \%$ Triton X-100), and then cell pellets were kept on ice for $20 \mathrm{~min}$ (with frequent mixing). Next, nuclei from cells were precipitated by centrifugation at 2,500 $\mathrm{g}$ for $15 \mathrm{~min}$, and then re-suspended in freshly RIP lysis buffer $(10 \mathrm{mM}$ HEPES-KOH $\mathrm{pH} 7,150 \mathrm{mM} \mathrm{KCl}, 5 \mathrm{mM} \mathrm{MgCl}$, $5 \mathrm{mM}$ EDTA, 0.5\% IGEPAL-CA-630, $0.5 \mathrm{mM}$ dithiothreitol, $0.2 \mathrm{mg} / \mathrm{mL}$ Heparin, $100 \mathrm{U} / \mathrm{mL}$ RNase OUT, $100 \mathrm{U} / \mathrm{mL}$ Superase IN, protease inhibitor tablet adding before use). After that, chromatin shearing was performed with sonication. The suspension was centrifuged at $14,000 \mathrm{~g}$ at $4^{\circ} \mathrm{C}$ for $10 \mathrm{~min}$, and the supernatant was harvested. Next, the nuclear extracts were precleared with rabbit IgG (Sigma) and Dynabeads ${ }^{\mathrm{m}}{ }^{\mathrm{m}}$ Protein G (Thermo Scientific). Next, the nuclei extracts were incubated with $5 \mu$ g antibodies against LSD1 (Millipore, 07-705), Setdla (Bethyl, A300-289A), and MLL1 (Novus Biologicals,
NB600-248) overnight at $4{ }^{\circ} \mathrm{C}$. Immuno-complexes were captured by incubating with $50 \mu \mathrm{l}$ Dynabeads ${ }^{\mathrm{Tw}}$ Protein G (Thermo Scientific) for another $2 \mathrm{~h}$ at $4^{\circ} \mathrm{C}$. Next, the precipitant was washed with the ice-cold washing buffer $(50 \mathrm{mM}$ Tris- $\mathrm{HCl} \mathrm{pH}$ 7.5, $150 \mathrm{mM} \mathrm{NaCl}, 1 \mathrm{mM} \mathrm{MgCl}$, 0.05\% IGEPAL-CA-630) supplemented with $0.02 \mathrm{mg} / \mathrm{mL}$ heparin. The RNA-protein complexes were eluted with $500 \mu \mathrm{L}$ elution buffer (50 mM Tris pH 8.0, $100 \mathrm{mM} \mathrm{NaCl}, 10 \mathrm{mM}$ EDTA, 1\% SDS) for $10 \mathrm{~min}$ at $65^{\circ} \mathrm{C}$. The precipitated RNA was extracted and purified by TRIzol RNA extraction reagent, eluted with nuclease-free water, treated with DNaseI, and then endogenous RNA was subjected to the RT-qPCR analysis.

Chromatin immunoprecipitation (ChIP). ChIP assay was performed as described previously ${ }^{42}$. Briefly, cells were cross-linked with $1 \%$ formaldehyde for $10 \mathrm{~min}$ at room temperature, and then quenched by addition of $125 \mathrm{mM}$ glycine for $5 \mathrm{~min}$ at room temperature. The cell pellets were washed with ice-cold 1x PBS. Then, the cell pellets were re-suspended in ChIP lysis buffer $(50 \mathrm{mM}$ Tris-HCl, $\mathrm{pH} 8,10 \mathrm{mM}$ EDTA, 1\% SDS). The lysates were sonicated with the Bioruprtor ${ }^{\text {min }}$ UCD200. After fragmentation, the lysates were centrifuged at $14,000 \mathrm{rpm}$ for $10 \mathrm{~min}$ at $4^{\circ} \mathrm{C}$. The supernatant was diluted with ChIP buffer $(20 \mathrm{mM}$ Tris- $\mathrm{HCl}$, pH 8.0, $2 \mathrm{mM}$ EDTA, $1 \%$ Triton X-100, $150 \mathrm{mM} \mathrm{NaCl}$ ). The $10 \%$ of remaining lysate was used as an input control. Sheared chromatin samples from $5 \times 10^{6}$ AML cells were immunoprecipitated with $5 \mu \mathrm{g}$ anti-MLL1 (Novus Biologicals, cat\# NB600-248) or $2.5 \mu \mathrm{g}$ antiH3K4me3 (Millipore, cat $\# 04-745$ ) overnight at $4^{\circ} \mathrm{C}$, separately. Then $50 \mu \mathrm{L}$ Dynabeads ${ }^{\text {mt }}$ Protein G (Thermo Fisher Scientific) was incubated with each ChIP sample for $2 \mathrm{~h}$ at $4{ }^{\circ} \mathrm{C}$ with rotation. The precipitated complexes were washed with low salt (20 mM Tris, pH 8.0, $150 \mathrm{mM} \mathrm{NaCl}, 2 \mathrm{mM}$ EDTA, $1 \%$ Triton X-100, 0.1\% SDS), high salt (20 mM Tris, pH 8.0, $500 \mathrm{mM} \mathrm{NaCl}, 2 \mathrm{mM}$ EDTA, $1 \%$ Triton X-100, 0.1\% SDS) and lithium chloride (10 mM Tris, pH 8.0, $1 \mathrm{mM}$ EDTA, $1 \%$ Triton X-100, $250 \mathrm{mM}$ $\mathrm{LiCl}, 1 \%$ sodium deoxycholate) washing buffer, with an additional final wash in TE buffer (50 mM Tris-HCl, pH 8.0, $10 \mathrm{mM}$ EDTA). Then, elution buffer (100 mM $\mathrm{NaHCO}_{3}, 1 \% \mathrm{SDS}$ ) was used to dissolve these precipitated complexes, and then were subjected to reverse crosslinking with $2 \mu \mathrm{L}$ of $10 \mathrm{mg} / \mathrm{mL}$ proteinase $\mathrm{K}$ overnight at $65^{\circ} \mathrm{C}$. After reverse-crosslinking, the DNA samples were purified and then analyzed by RT-qPCR. The final results represent the percentage of input chromatin and error bars through triplicate experiments. The MLL1 and H3K4me3 ChIP-DNA libraries were prepared using Illumina's TruSeq ChIP Sample Preparation Kit according to the manufacturer's instructions (Cat\# IP-202-1012). In brief, $10 \mathrm{ng}$ ChIP DNA fragments were performed end repair using the End Repair Mix, and then purified with the AMPure XP beads. After that, $3^{\prime}$ ends of the ChIP DNA fragments were adenylated with the A-Tailing Mix, and then ligated with the adapter indices. After that, ChIP DNA fragments were amplified with the adapter primers. Then, the quality of these DNA libraries were tested with the Qubit and Agilent Bioanalyzer. Final libraries were submitted to paired-end sequencing of 50 bp length on an Illumina HiSeq 3000 The quality of the library was checked with Qubit and Agilent Bioanalyzer. Final libraries were submitted to paired-end sequencing of $100 \mathrm{bp}$ length on an Illumina HiSeq 3000.

Chromatin Isolation by RNA Immunoprecipitation (ChIRP) assay. ChIRP assay was carried out to analyze the HoxBlinc lncRNA distribution in the genome according to our previously described with some modifications ${ }^{43}$. Briefly, 20 million cells were harvested and cross-linked in $20 \mathrm{ml}$ of PBS buffer containing $1 \%$ glutaraldehyde (Sigma, Cat\# G5882) at room temperature for $10 \mathrm{~min}$, and then were quenched with a $1 / 10$ th volume of $1.25 \mathrm{M}$ glycine at room temperature for $5 \mathrm{~min}$. Next, these cross-linked cells were washed twice with chilled PBS, and per $100 \mathrm{mg}$ of the pellet of cells were lysed in $1 \mathrm{ml}$ lysis buffer $(50 \mathrm{mM}$ Tris- $\mathrm{HCl} \mathrm{pH} \mathrm{7.0,}$ $10 \mathrm{mM}$ EDTA, 1\% SDS, PMSF, DTT, P.I. and SUPERase were added before use) Then, these lysates were sonicated $30 \mathrm{~min}$ using a Bioruptor (Diagenode) to prepare chromatin in a $4^{\circ} \mathrm{C}$ water bath at the highest setting with $30 \mathrm{~s} \mathrm{ON}, 30 \mathrm{~s}$ OFF pulse intervals. Next, the sheared chromatin was centrifuged and supernatant was diluted using hybridization buffer $(750 \mathrm{mM} \mathrm{NaCl}, 1 \%$ SDS, $50 \mathrm{mM}$ Tris- $\mathrm{HCl} 7.0$, $1.0 \mathrm{mM}$ EDTA, 15\% Formamide, add DTT, PMSF, P.I, and SUPERase-in fresh). After that, the diluted supernatant was hybridized with 100 pmol of biotinylated DNA probes targeting HoxBlinc or LacZ (sequences are listed in Supplementary Table 6) and incubated for $4 \mathrm{~h}$ at $37^{\circ} \mathrm{C}$ with shaking, and then added $100 \mu \mathrm{l}$ of Streptavidin-magnetic $\mathrm{C} 1$ beads (Invitrogen) for each sample for $30 \mathrm{~min}$ at $37^{\circ} \mathrm{C}$ Then, these precipitant were washed 5 times with washing buffer (2x SSC, $0.5 \%$ SDS). RNA fraction was extracted from ChIRP samples with TRIzol reagent and then subjected to analyze the HoxBlinc retrieval by RT-qPCR, and $\beta$-actin gene was used as a negative control. DNA fraction was extracted from ChIRP samples with Phenol:Chloroform:Isoamyl Alcohol (25:24:1, v/v), and then precipitated with $3 \mu \mathrm{L}$ of $20 \mathrm{mg} / \mathrm{mL}$ glycogen, $1 / 10^{\text {th }}$ volume of $3 \mathrm{M}$ sodium acetate ( $\mathrm{pH} 5.2$ ), and 2.5 volumes of $100 \%$ ethanol. Then ChIRP-DNA library was prepared for ChIRP-seq Libraries were constructed using Illumina's TruSeq ChIP Sample Preparation Kit according to the manufacturer's instructions (Cat\# IP-202-1012). In brief, $10 \mathrm{ng}$ ChIRP DNA fragments were performed end repair using the End Repair Mix, and then purified with AMPure XP beads. After that, 3 ' ends of the ChIRP DNA fragments were adenylated with A-Tailing Mix, and then ligated with the adapter indices. After that, ChIRP DNA fragments were amplified with the adapter primers. The quality of these ChIRP DNA libraries were examined with Qubit and Agilent Bioanalyzer. Final ChIRP libraries were submitted to the paired-end sequencing of $100 \mathrm{bp}$ length on an Illumina HiSeq 2500. 
ChIP-seq and ChIRP-seq data analysis. The cutadapt (http://cutadapt. readthedocs.io, version 1.2.0) program was used to trim the adaptors and low quality reads from the ChIP-seq or ChIRP-seq raw data ${ }^{33}$. These Cutadapt-filtered reads were aligned to mouse reference genome $(\mathrm{mm} 9)$ using Bowtie 2 with default parameters ${ }^{35}$, and the quality of these trimmed data was evaluated by the FastQC program $^{44}$. After alignment, samtools program was employed to convert the SAM files into BAM files and sorted these BAM files ${ }^{45}$. Next, peak calling was performed using peak calling algorithm MACS2 $2^{46}$. Peaks were transformed to the visualized files (bigwig format) with deepTools ${ }^{47}$, including control and experimental datasets. All sequencing tracks were visualized using the Integrated Genomic Viewer software (IGV) ${ }^{48}$. Peaks were annotated with"annotatePeaks.pl" program with HOMER package ${ }^{49}$. Differential Peaks calling was performed with getDifferentialPeaks program in HOMER software. For ChIRP-seq binding motif analysis, the de novo motif analysis was performed by the "findmotifsgenome.pl" from the HOMER motif discovery algorithm ${ }^{49}$. The HoxBlinc bound regions associated genes and pathways were analyzed and annotated by the Gene Ontology (GO) analysis with the Database for Annotation, Visualization and Integrated Discovery (DAVID) tool (https://david.ncifcrf.gov/, Version 6.8)**37. Each GO term with a p-value more than $1 \times 10^{-3}$ is used for cutoff (threshold: $10^{-3}$ ). All genomics datasets were deposited in the NCBI GEO under accession number (GSE115096).

Circular Chromosome conformation capture (4C) assays. The 4C-seq assay was performed as previously described ${ }^{50}$ with minor modifications. In brief, $2 \times 10^{6}$ cells were cross-linked with $1 \%$ formaldehyde for $10 \mathrm{~min}$ and the reaction was quenched with $0.125 \mathrm{M}$ glycine for $5 \mathrm{~min}$ at room temperature. Cells were washed twice with cold $\mathrm{PBS}$, and re-suspended in the digestion buffer containing $0.3 \%$ SDS overnight at $37^{\circ} \mathrm{C}$ with shaking. After that, $2 \%$ Triton X-100 was added to sequester SDS and incubated for $1.5 \mathrm{~h}$ at $37^{\circ} \mathrm{C}$ with shaking. Then, the chromatin was digested with $400 \mathrm{U}$ of BglII enzyme (NEB) at $37^{\circ} \mathrm{C}$ overnight. $1.6 \%$ SDS buffer was added to stop the digestion at $65^{\circ} \mathrm{C}$ for $20 \mathrm{~min}$. After that, the digested chromatin was diluted in T4 DNA ligation buffer (NEB) containing $1 \%$ Triton X-100 and incubated at $37^{\circ} \mathrm{C}$ for $1.5 \mathrm{~h}$ with shaking. Next, 800U of T4 DNA ligase (NEB) was used to ligate these digested chromatin at $16^{\circ} \mathrm{C}$ for 3 days followed by $1 \mathrm{hr}$ at room temperature. Then, reverse crosslinking was carried out by adding $200 \mu \mathrm{g}$ of Proteinase K (Invitrogen) and incubated at $65^{\circ} \mathrm{C}$ overnight. Next, the $3 \mathrm{C}$ DNA was subjected to phenol:chloroform and extracted with $100 \%$ ethanol, and then dissolved with $\mathrm{ddH}_{2} \mathrm{O}$. For the second digestion, the first ligates was digested with $300 \mathrm{U}$ of NlaIII (NEB) at $37^{\circ} \mathrm{C}$ overnight with shaking. The reaction was stopped by adding SDS to a final concentration of $1.6 \%$ at $65^{\circ} \mathrm{C}$ for 20 $\mathrm{min}$. The digested chromatin/DNA was ligated in ligation buffer containing $6000 \mathrm{U}$ of T4 DNA ligase at $16^{\circ} \mathrm{C}$ overnight. After ligation, the $4 \mathrm{C}$ DNA was extracted by phenolchloroform, and purified by Qiagen PCR kit and amplified by inverse PCR using baitspecific primers. The invert PCR products were cloned into PGEM $^{\oplus}-\mathrm{T}$ Easy Vector Systems (Promega) for Sanger sequencing. 4C-seq Libraries were constructed by adding barcoded Illumina adapters to the $5^{\prime}$ end of each primer (Supplementary Table 6). PCR reactions were performed using the Expand Long Template PCR System (Roche), and DNA libraries were purified and quantified before sequencing. The bar-coded DNA libraries were sequenced as 150 bp pair-end reads using the Illumina Nextseq500 platform. For data analysis, cutadapt (http://cutadapt.readthedocs.io, version 1.2.0) program was carried out to trim the $4 \mathrm{C}$-seq raw data and remove the adaptors and low quality reads ${ }^{33}$. Filtered reads were aligned to the reference mouse genome (build $\mathrm{mm} 9$ ) with Bowtie2 2.2.951. 4C-seq data was analyzed using the 4cseq_pipeline ${ }^{52}$ and normalized using DESeq $2^{53}$. Statistical analysis for differential interactions between genotypes was performed using DESeq2. Spearman correlation of each genotype was performed using $\mathrm{R}^{54}$. The $4 \mathrm{C}$-sequencing sequence reads have been deposited in the NCBI GEO database (GSE115096).

\section{Transposase-Accessible Chromatin using sequencing (ATAC-seq) assay.} ATAC-seq assay was used to analyze the genome chromatin accessibility in different experimental conditions as described previously ${ }^{12,55}$. In Brief, $5 \times 10^{4}$ cells were collected for library preparation. Cells were washed with pre-cold phosphate buffered saline (PBS) and re-suspended in lysis buffer containing $10 \mathrm{mM}$ Tris- $\mathrm{HCl}$ (pH 7.4), $10 \mathrm{mM} \mathrm{NaCl}, 3 \mathrm{mM} \mathrm{MgCl} 2,0.1 \%$ NP-40. After washing with cold 1x PBS buffer, the cell pellets were re-suspended in fragment buffer and then fragmented with $\mathrm{Tn} 5 \mathrm{Transposes}$ for transposition reaction at $37^{\circ} \mathrm{C}$ for $30 \mathrm{~min}$. Then these DNA fragments were extracted and purified using the MinElute Kit (QIAGEN). The preparation of library fragments were amplified using $1 \mathrm{x}$ NEB next PCR master mix and $1.25 \mu \mathrm{M}$ indexed Nextra PCR primers (Supplementary Table 6) with following PCR conditions: $72^{\circ} \mathrm{C}$ for $5 \mathrm{~min}, 98^{\circ} \mathrm{C}$ for $30 \mathrm{~s}$, followed by thermocycling at $98^{\circ} \mathrm{C}$ for $10 \mathrm{~s}, 63^{\circ} \mathrm{C}$ for $30 \mathrm{~s}$ and $72^{\circ} \mathrm{C}$ for $1 \mathrm{~min}$. After amplification, the eluted DNA was used in a quantitative PCR (qPCR) reaction to estimate the optimum number of amplification cycles. Libraries were quantified using qPCR (Kapa Library Quantification Kit for Illumina, Roche), and AMPure XP beads (Beckman Coulter) was employed to purify the libraries, and then the quality of the DNA library was examined by Agilent Bioanalyzer 2100 prior to sequencing with $2 \times 100$ bp paired-end reads on an Illumina HiSeq 2500. Each sample includes two replicates for statistical analysis.

ATAC-seq analysis. ATAC-seq assay was carried out and analyzed with two biological replicates according to our previous reports ${ }^{12,55}$. Briefly, all of the raw data files were filtered through cutadapt (http://cutadapt.readthedocs.io, version 1.2.0) to remove adaptors and low quality reads ${ }^{33}$. These filtered reads were aligned to the mouse genome $(\mathrm{mm} 9)$ using Bowtie2 with default parameters (version Bowtie $2 / 2.2 .6)^{35}$, and the quality of these trimmed data was evaluated by FastQC program (version 0.11 .8$)^{44}$. PCR duplicates were removed using Picard MarkDuplicates (version 2.0.1), and mitochondrial reads were removed with samtools ${ }^{56}$. ENCODE blacklist regions were filtered (https://sites.google.com/site/ anshulkundaje/projects/blacklists). For quality control, 50 million reads with paired-end sequencing was used for each sample. In addition, the alignment rate of each replicate is more than $95 \%$ through removing the unaligned reads. Third, the mitochondrial-related reads and PCR duplicates reads were removed from total reads after alignment. Finally, non-uniquely aligned reads were filtered based on MAPQ scores with samtools (MAPQ > 30), and plotPCA from BiocGenerics package in $\mathrm{R}$ package (R/3.6.1) was carried out to identify the variance between control and experimental groups. Moreover, fragSizeDist from ATACseqQC package in $\mathrm{R}$ package was used to show the fragment size distribution in control and experimental groups. After alignment and trimming, samtools (version 1.8.0) were used to convert the SAM files into BAM files and sorted the BAM files for further analysis ${ }^{45}$. Peak calling was performed using peak calling algorithm MACS2 with parameters ("-g mm -p 1e-9 -nolambda -f BAMPE -nomodel -shiftsize $=100$ --extsize $200 "{ }^{46}$. The visualizable bigWig files of fragment or read coverages were generated with bedGraphToBigWig program, including control and experimental datasets (https://www.encodeproject.org/software/bedgraphtobigwig/). All sequencing tracks were viewed using the Integrated Genomic Viewer (IGV/2.4.19) ${ }^{48}$. Peaks were annotated with the command "annotatePeaks.pl" from HOMER package (version 4.10$)^{49}$ and GREAT ${ }^{57}$. Next, the differential binding sites between two peak files were calculated with DEseq2 (Benjamini-Hochberg adjusted $p<0.05$; FoldChange $\geq 2$ ), including control and experimental groups with $C+G$ normalized and "reads in peaks" normalized data ${ }^{58}$. The de novo motif analysis was performed by the "findmotifsgenome.pl" from the HOMER package ${ }^{99}$. For each genomic feature (peaks or chromVAR annotation), the chromatin accessibility median deviation z-score (for chromVAR features) or fragment counts (for peaks) were examined in control and experimental groups with chromVAR package in $\mathrm{R}$ language ${ }^{59,60}$. Overall similarity between the replicates of ATAC-seq global chromatin accessibility signatures was carried out with Pearson's correlation coefficient and Pearson's $\chi^{2}$-test. All genomics datasets were deposited in the NCBI GEO under accession number (GSE115096).

dCas9-mediated inactivation of HoxBlinc in AML cells. To generate the CRISPRi sgRNA vector, sgRNA targeting the promoters of HOXBLINC were designed using the Zhang laboratory web tool (http://crispr.mit.edu), and sgRNA was subcloned into the pLKO5.sgRNA.EFS.GFP vector (Addgene\#57822). OCI-AML3 AML cells were co-transfected with the gRNA plasmids encoding GFP and the repressive plasmid encoding dCas9-KRAB (pHR-SFFV-dCas9-BFP-KRAB, Addgene plasmid \#46911), and then these cells were cultured for another $48 \mathrm{~h}$. After that, OCI-AML3 AML cells were subjected to $2 \mu \mathrm{g} / \mathrm{mL}$ of puromycin for another $48 \mathrm{~h}$, and then live cells were sorted with GFP by FACS. Finally, RNAs from these positive cells were extracted with TRIzol $^{\mathrm{m}}{ }^{\mathrm{x}}$ reagent, and then the gene expression level was determined by RT-qPCR.

Doxycycline (DOX) inducible shHOXBLINC in AML cells. Human shRNA targeting HOXBLINC was designed using the Thermo Fisher web tool (https:// rnaidesigner.thermofisher.com/rnaiexpress/), and shRNA was cloned into the doxycycline (DOX) inducible pTRIPz vector (dharmacon.horizondiscovery.com). Then OCI-AML3 cells were infected with DOX inducible pTRIPz vector and then cultured for $48 \mathrm{~h}$. After that, cells were selected with $2 \mu \mathrm{g} / \mathrm{ml}$ of puromycin for another $48 \mathrm{~h}$, and the live cells were cultured in the standard culture medium of OCI-AML3 cell line. After that, cells were treated with the DOX at a concentration of $2 \mu \mathrm{g} / \mathrm{mL}$ for 3 days. In addition, refresh or add the fresh culture medium to maintain the doxycycline concentration every $48 \mathrm{~h}$. Then, RNAs from these doxycycline inducible cells were extracted and purified with the TRIzol ${ }^{\text {mex }}$ reagent, and then proceeded to evaluate the knockdown efficiency by RT-qPCR

Lentiviral transduction. The RNAi Consortium (TRC)-based short hairpin RNA lentiviral vector (Sigma, Horizon Discovery, or lab-owned) were transfected into HEK 293 T cells (ATCC) together with pMD2.G (Addgene) and psPAX2 (Addgene) to package viruses. The virus supernatant was collected from $16 \mathrm{~h}$ to $48 \mathrm{~h}$ after transfection and then purified with Lenti-X ${ }^{\mathrm{x}}$ Concentrator (Takara). The Lin ${ }^{-}$cells from mice BMs were transduced with the packaged viruses for $24 \mathrm{~h}$ in plain IMDM added with $\mathrm{mSCF}(100 \mathrm{ng} / \mathrm{mL})$ and then sorted for $\mathrm{GFP}^{+}$LSK cells. Sorted LSK cells were collected into different dishes for colony-forming assays or transplantation.

Xenotransplantation of OCI-AML3 AML cells or AML patient BM cells. Adult NOD.Cg-Prkdc $c^{\text {scid }} I l 2 r g^{\mathrm{tm} 1 \mathrm{Wjl}} / \mathrm{SzJ}$ (NSG) mice (6-8 weeks old) were pretreated with 280 cGy total body irradiation, then $5 \times 10^{5}$ viable OCI-AML3 cells (in $300 \mu \mathrm{l}$ PBS) or $1 \times 10^{6}$ patient BM cells (in $300 \mu \mathrm{PBS}$ ) were injected into tail veins of the recipient NSG mice for transplantation. After transplantation, the recipients mice were administered with (for OCI-AML3 cells stably expressing inducible shHOXBLINC to induce HoxBlinc KD) or without (for AML cells with dCas9-mediated HoxBlinc inactivation) doxycycline in the drinking water (Sigma D-9891, 1 mg/ml, 
$1 \%$ sucrose, newly prepared every other day) until being sacrificed, and daily monitored for symptoms (ruffled coat, hunched back, weakness, and reduced motility) and survival time. For each set of xenotransplantation, recipient mice of all groups were killed and analyzed on the same day when any group of recipients exhibited a moribund condition. Human CD45 chimerism in the BM, spleen cells, and PB WBC were analyzed by flow cytometry, histological assay, IHC for hCD45 were also performed as described above.

Quantification and statistical analysis. Differences between experimental groups were determined by the Student's $t$-test or analysis of variance (ANOVA) followed by Newman-Keuls multiple comparison tests as appropriate. $P<0.05$ is considered significant. For in vivo experiments, the sample size chosen was based on the generalized linear model with Bonferroni multiple comparison adjustments; with the proposed sample size of at least five mice/group/genotype. Animals were randomly assigned to each study. For all in vitro experiments, at least three independent experiments with more than three biological replicates for each condition/genotype were performed to ensure adequate statistical power.

Reporting summary. Further information on research design is available in the Nature Research Reporting Summary linked to this article.

\section{Data availability}

Sequencing data of RNA-seq, ATAC-seq, ChIP-seq and 4C-seq in Figs. 1, 4, 5, 6 \& Supplementary Figs. 1, 2, 6, 7, 9 were deposited in the Gene Expression Omnibus (accession number GSE115096). RNA-seq data of AML patient samples were retrieved from The Cancer Genome Atlas (GEO accession number GSE62944). NCBI GEO public database (GSM918748) were used for CTCF binding site analysis of Fig. 5 and Supplementary Fig. 7. Source Data file is provided with this paper. All biological material were either directly commercially available or are available upon request from the lab (see Supplementary Table 7). Supplemental Information Supplemental information includes 9 figures and 9 tables. All other data are available from the authors. Source data are provided with this paper.

\section{Code availability}

There is no custom R code in this study. The source of software and algorithms are included in Supplementary Table 7.

Received: 25 July 2020; Accepted: 24 February 2021; Published online: 29 March 2021

\section{References}

1. Falini, B. et al. Cytoplasmic nucleophosmin in acute myelogenous leukemia with a normal karyotype. N. Engl. J. Med. 352, 254-266 (2005).

2. Schlenk, R. F. et al. Mutations and treatment outcome in cytogenetically normal acute myeloid leukemia. N. Engl. J. Med. 358, 1909-1918 (2008).

3. Arber, D. A. et al. The 2016 revision to the World Health Organization classification of myeloid neoplasms and acute leukemia. Blood 127, 2391-2405 (2016).

4. Grisendi, S. et al. Role of nucleophosmin in embryonic development and tumorigenesis. Nature 437, 147-153 (2005).

5. Falini, B. et al. Immunohistochemistry predicts nucleophosmin (NPM) mutations in acute myeloid leukemia. Blood 108, 1999-2005 (2006).

6. Bolli, N. et al. Born to be exported: $\mathrm{COOH}$-terminal nuclear export signals of different strength ensure cytoplasmic accumulation of nucleophosmin leukemic mutants. Cancer Res. 67, 6230-6237 (2007).

7. Alcalay, M. et al. Acute myeloid leukemia bearing cytoplasmic nucleophosmin (NPMc+ AML) shows a distinct gene expression profile characterized by upregulation of genes involved in stem-cell maintenance. Blood 106, 899-902 (2005).

8. Spencer, D. H. et al. Epigenomic analysis of the HOX gene loci reveals mechanisms that may control canonical expression patterns in AML and normal hematopoietic cells. Leukemia 29, 1279-1289 (2015).

9. Alharbi, R. A., Pettengell, R., Pandha, H. S. \& Morgan, R. The role of HOX genes in normal hematopoiesis and acute leukemia. Leukemia 27, 1000-1008 (2013).

10. Deng, C. et al. HoxBlinc RNA recruits Set1/MLL complexes to activate Hox gene expression patterns and mesoderm lineage development. Cell Rep. 14, 103-114 (2016).

11. Wang, K. C. et al. A long noncoding RNA maintains active chromatin to coordinate homeotic gene expression. Nature 472, 120-124 (2011).

12. Luo, H. et al. HOTTIP lncRNA promotes hematopoietic stem cell self-renewal leading to AML-like disease in mice. Cancer Cell 36, 645-659 e648 (2019).

13. Mallardo, M. et al. NPMc+ and FLT3_ITD mutations cooperate in inducing acute leukaemia in a novel mouse model. Leukemia 27, 2248-2251 (2013).
14. Vassiliou, G. S. et al. Mutant nucleophosmin and cooperating pathways drive leukemia initiation and progression in mice. Nat. Genet. 43, 470-475 (2011).

15. Luo, H. et al. CTCF boundary remodels chromatin domain and drives aberrant HOX gene transcription in acute myeloid leukemia. Blood, https:// doi.org/10.1182/blood-2017-11-814319 (2018).

16. Brunetti, L. et al. Mutant NPM1 maintains the leukemic state through HOX expression. Cancer Cell 34, 499-512 e499 (2018).

17. Kuhn, M. W. et al. Targeting chromatin regulators inhibits leukemogenic gene expression in NPM1 mutant leukemia. Cancer Disco. 6, 1166-1181 (2016).

18. Huarte, M. The emerging role of lncRNAs in cancer. Nat. Med. 21, 1253-1261 (2015).

19. Trimarchi, T. et al. Genome-wide mapping and characterization of Notchregulated long noncoding RNAs in acute leukemia. Cell 158, 593-606 (2014).

20. Garzon, R. et al. Expression and prognostic impact of lncRNAs in acute myeloid leukemia. Proc. Natl Acad. Sci. USA 111, 18679-18684 (2014).

21. Schwarzer, A. et al. The non-coding RNA landscape of human hematopoiesis and leukemia. Nat. Commun. 8, 218 (2017).

22. Luo, M. et al. Long non-coding RNAs control hematopoietic stem cell function. Cell Stem Cell 16, 426-438 (2015).

23. Batista, P. J. \& Chang, H. Y. Long noncoding RNAs: cellular address codes in development and disease. Cell 152, 1298-1307 (2013).

24. Li, Y. et al. Setdla and NURF mediate chromatin dynamics and gene regulation during erythroid lineage commitment and differentiation. Nucleic Acids Res. 44, 7173-7188 (2016).

25. Arndt, K. et al. SETD1A protects HSCs from activation-induced functional decline in vivo. Blood 131, 1311-1324 (2018)

26. Falini, B. et al. NPM1 mutations and cytoplasmic nucleophosmin are mutually exclusive of recurrent genetic abnormalities: a comparative analysis of 2562 patients with acute myeloid leukemia. Haematologica 93, 439-442 (2008).

27. Cancer Genome Atlas Research, N. et al. Genomic and epigenomic landscapes of adult de novo acute myeloid leukemia. N. Engl. J. Med. 368, 2059-2074 (2013).

28. Qian, P. et al. Retinoid-sensitive epigenetic regulation of the Hoxb cluster maintains normal hematopoiesis and inhibits leukemogenesis. Cell Stem Cell 22, 740-754 e747 (2018).

29. Gaidzik, V. I. et al. RUNX1 mutations in acute myeloid leukemia: results from a comprehensive genetic and clinical analysis from the AML study group. J. Clin. Oncol. 29, 1364-1372 (2011)

30. Yusufzai, T. M., Tagami, H., Nakatani, Y. \& Felsenfeld, G. CTCF tethers an insulator to subnuclear sites, suggesting shared insulator mechanisms across species. Mol. Cell 13, 291-298 (2004).

31. Yang, H. et al. Gain of function of ASXL1 truncating protein in the pathogenesis of myeloid malignancies. Blood 131, 328-341 (2018).

32. Li, J. et al. Loss of Asxl2 leads to myeloid malignancies in mice. Nat. Commun. 8, 15456 (2017).

33. Trapnell, C. et al. Differential gene and transcript expression analysis of RNA seq experiments with TopHat and Cufflinks. Nat. Protoc. 7, 562-578 (2012)

34. Langmead, B., Trapnell, C., Pop, M. \& Salzberg, S. L. Ultrafast and memoryefficient alignment of short DNA sequences to the human genome. Genome Biol. 10, R25 (2009).

35. Trapnell, C., Pachter, L. \& Salzberg, S. L. TopHat: discovering splice junctions with RNA-Seq. Bioinformatics 25, 1105-1111 (2009).

36. Trapnell, C. et al. Transcript assembly and quantification by RNA-Seq reveals unannotated transcripts and isoform switching during cell differentiation. Nat. Biotechnol. 28, 511-515 (2010).

37. Huang, D. W., Sherman, B. T. \& Lempicki, R. A. Systematic and integrative analysis of large gene lists using DAVID bioinformatics resources. Nat. Protoc. 4, 44-57 (2009)

38. Subramanian, A. et al. Gene set enrichment analysis: a knowledge-based approach for interpreting genome-wide expression profiles. Proc. Natl Acad. Sci. USA 102, 15545-15550 (2005).

39. Tsai, M. C. et al. Long noncoding RNA as modular scaffold of histone modification complexes. Science 329, 689-693 (2010).

40. Deng, C. et al. HoxBlinc RNA recruits Set1/MLL complexes to activate Hox gene expression patterns and mesoderm lineage development. Cell Rep. 14, 103-114 (2016).

41. Deng, C. et al. USF1 and hSET1A mediated epigenetic modifications regulate lineage differentiation and HoxB4 transcription. PLoS Genet. 9, e1003524 (2013)

42. Chu, C., Qu, K., Zhong, F. L., Artandi, S. E. \& Chang, H. Y. Genomic maps of long noncoding RNA occupancy reveal principles of RNA-chromatin interactions. Mol. Cell 44, 667-678 (2011).

43. Martin, M. Cutadapt removes adapter sequences from high-throughput sequencing reads. EMBnet J. 17, 10-12 (2011).

44. Wingett, S. W. \& Andrews, S. FastQ Screen: a tool for multi-genome mapping and quality control. F1000Res 7, 1338 (2018).

45. Li, H. et al. The sequence alignment/Map format and SAMtools. Bioinformatics 25, 2078-2079 (2009).

46. Zhang, Y. et al. Model-based analysis of ChIP-Seq (MACS). Genome Biol. 9 , R137 (2008). 
47. Ramirez, F. et al. deepTools2: a next generation web server for deepsequencing data analysis. Nucleic Acids Res. 44, W160-W165 (2016).

48. Robinson, J. T. et al. Integrative genomics viewer. Nat. Biotechnol. 29, 24-26 (2011).

49. Heinz, S. et al. Simple combinations of lineage-determining transcription factors prime cis-regulatory elements required for macrophage and B cell identities. Mol. Cell 38, 576-589 (2010).

50. Stadhouders, R. et al. Multiplexed chromosome conformation capture sequencing for rapid genome-scale high-resolution detection of long-range chromatin interactions. Nat. Protoc. 8, 509-524 (2013).

51. Langmead, B. \& Salzberg, S. L. Fast gapped-read alignment with Bowtie 2. Nat. Methods 9, 357-359 (2012).

52. van de Werken, H. J. et al. Robust $4 \mathrm{C}$-seq data analysis to screen for regulatory DNA interactions. Nat. Methods 9, 969-972 (2012).

53. Love, M. I., Huber, W. \& Anders, S. Moderated estimation of fold change and dispersion for RNA-seq data with DESeq2. Genome Biol. 15, 550 (2014).

54. Huang, Y. et al. cis-Regulatory circuits regulating NEK6 kinase overexpression in transformed B cells are super-enhancer independent. Cell Rep. 18, 2918-2931 (2017).

55. Buenrostro, J. D., Wu, B., Chang, H. Y. \& Greenleaf, W. J. ATAC-seq: a method for assaying chromatin accessibility genome-wide. Curr. Protoc. Mol. Biol. 109, 2129 21-21 2929 (2015).

56. Corces, M. R. et al. An improved ATAC-seq protocol reduces background and enables interrogation of frozen tissues. Nat. Methods 14, 959-962 (2017).

57. McLean, C. Y. et al. GREAT improves functional interpretation of cisregulatory regions. Nat. Biotechnol. 28, 495-501 (2010).

58. Ross-Innes, C. S. et al. Differential oestrogen receptor binding is associated with clinical outcome in breast cancer. Nature 481, 389 (2012).

59. Schep, A. N., Wu, B., Buenrostro, J. D. \& Greenleaf, W. J. chromVAR: inferring transcription-factor-associated accessibility from single-cell epigenomic data. Nat. Methods 14, 975-978 (2017).

60. Rubin, A. J. et al. Coupled single-cell CRISPR screening and epigenomic profiling reveals causal gene regulatory networks. Cell 176, 361-376 e317 (2019).

\section{Acknowledgements}

This work was supported by grants from the National Institute of Health (R01HL141950 to M.X. and S.H., R01CA172408 to M.X. and F-C.Y., NIH R00CA178191 and R01DK121831 to O.A.G., and R01DK110108 to S.H.), the Cancer Prevention \& Research Institute of Texas (CPRIT RP200242 to M.X.), as well as the Florida Academic Cancer Center Alliance (FACCA) Award (S.H. and M.X.). We also thank Rachael Mills at the Penn State Hershey College of Medicine for editing the manuscript.

\section{Author contributions}

G.Z., H.L., Y.Q. F-C.Y., S.H., and M.X. conceived and designed experiments. G.Z., H.L S.C., Q.L., and Y.G. performed experiments. H.L. performed bioinformatics and statistical analysis for mouse NGS data. J.X., Z.Z., R.F., A.S., D.C., B.X., M.R., and W.L. acquired and analyzed primary human patient samples and performed TCGA AML data analysis. H.N analyzed the histological slides from mouse tissues. Y.F. and O.A.G. provided $\mathrm{Npmlc}^{+}$knock in mouse and related samples. M.X. and S.H. wrote the original draft, O.A.G., S.D.N., and F.-C.Y. revised and edited the manuscript.

\section{Competing interests}

The authors declare no competing interests.

\section{Additional information}

Supplementary information The online version contains supplementary material available at https://doi.org/10.1038/s41467-021-22095-2.

Correspondence and requests for materials should be addressed to S.H. or M.X.

Peer review information Nature Communications thanks Cheng Cheng Zhang and Dong-Er Zhang for their contribution to the peer review of this work. Peer reviewer reports are available.

Reprints and permission information is available at http://www.nature.com/reprints

Publisher's note Springer Nature remains neutral with regard to jurisdictional claims in published maps and institutional affiliations.

\begin{abstract}
Open Access This article is licensed under a Creative Commons Attribution 4.0 International License, which permits use, sharing, adaptation, distribution and reproduction in any medium or format, as long as you give appropriate credit to the original author(s) and the source, provide a link to the Creative Commons license, and indicate if changes were made. The images or other third party material in this article are included in the article's Creative Commons license, unless indicated otherwise in a credit line to the material. If material is not included in the article's Creative Commons license and your intended use is not permitted by statutory regulation or exceeds the permitted use, you will need to obtain permission directly from the copyright holder. To view a copy of this license, visit http://creativecommons.org/ licenses/by/4.0/.
\end{abstract}

(C) The Author(s) 2021

${ }^{1}$ Department of Molecular Medicine, University of Texas Health San Antonio, San Antonio, TX 78229, USA. ${ }^{2}$ Department of Biochemistry and Molecular Biology, University of Miami Miller School of Medicine, Miami, FL 33136, USA. ${ }^{3}$ Department of Pediatrics, Pennsylvania State University College of Medicine, Hershey, PA 17033, USA. ${ }^{4}$ Department of Pharmacology and Therapeutics, University of Florida College of Medicine, Gainesville, FL 32610, USA. ${ }^{5}$ Department of Molecular and Cellular Biology, Dan L. Duncan Cancer Center, Baylor College of Medicine, Houston, TX 77030, USA. ${ }^{6}$ Division of Hematology/Oncology, Department of Medicine, Pennsylvania State University College of Medicine, Hershey, PA 17033, USA. ${ }^{7}$ Department of Hematology, The First Affiliated Hospital of Xiamen University, Xiamen 361026 Fujian, China. ${ }^{8}$ Department of Hematology and Oncology, Tianjin Medical University Cancer Institute and Hospital, National Clinical Research Center for Cancer, Key Laboratory of Cancer Prevention and Therapy, Tianjin 300060, China. ${ }^{9}$ Department of Hematology, Nanfang Hospital, Southern Medical University, Guangzhou 510515 Guangdong, China. ${ }^{10}$ Department of Pathology, Wayne State University School of Medicine, Detroit, MI 48201, USA. ${ }^{11}$ Penn State Cancer Institute, Pennsylvania State University College of Medicine, Hershey, PA 17033, USA. ${ }^{12}$ Department of Cell System \& Anatomy, University of Texas Health San Antonio, San Antonio, TX 78229, USA. ${ }^{13}$ Department of Medicine, University of Texas Health San Antonio, San Antonio, TX 78229, USA. ${ }^{14}$ Mays Cancer Center, University of Texas Health San Antonio, San Antonio, TX 78229, USA. ${ }^{15}$ Department of Cellular \& Molecular Physiology, Pennsylvania State University College of Medicine, Hershey, PA 17033, USA. ${ }^{16}$ Sylvester Comprehensive Cancer Center, University of Miami Miller School of Medicine, Miami, FL 33136, USA. ${ }^{17}$ Division of Computational Biomedicine, Department of Biological Chemistry, School of Medicine, University of California, Irvine, CA 92697, USA. ${ }^{18}$ Department of Medicine, University of Miami Miller School of Medicine, Miami, FL 33136, USA. ${ }^{19}$ These authors contributed equally: Ganqian Zhu, Huacheng Luo. ${ }^{20}$ These authors

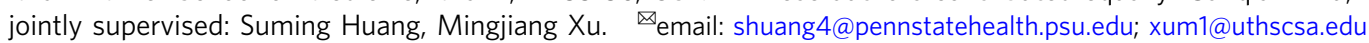

